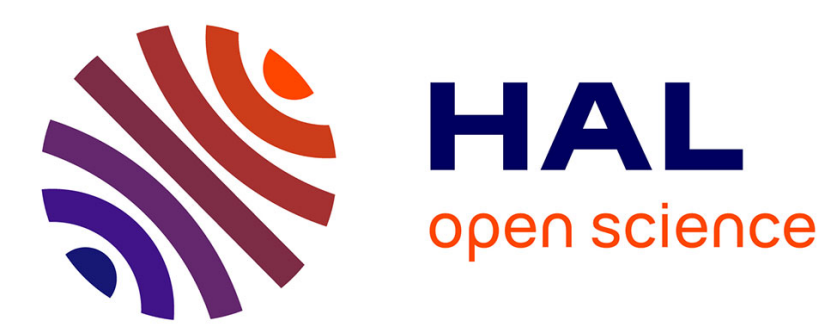

\title{
Apposition of the topological sensitivity and linear sampling approaches to inverse scattering
}

\author{
Cédric Bellis, Marc Bonnet, B. B. Guzina
}

\section{To cite this version:}

Cédric Bellis, Marc Bonnet, B. B. Guzina. Apposition of the topological sensitivity and linear sampling approaches to inverse scattering. Wave Motion, 2013, 50, pp.891-908. 10.1016/j.wavemoti.2013.02.013 . hal-00797649

\section{HAL Id: hal-00797649 \\ https://hal.science/hal-00797649}

Submitted on 7 Mar 2013

HAL is a multi-disciplinary open access archive for the deposit and dissemination of scientific research documents, whether they are published or not. The documents may come from teaching and research institutions in France or abroad, or from public or private research centers.
L'archive ouverte pluridisciplinaire HAL, est destinée au dépôt et à la diffusion de documents scientifiques de niveau recherche, publiés ou non, émanant des établissements d'enseignement et de recherche français ou étrangers, des laboratoires publics ou privés. 


\title{
Apposition of the topological sensitivity and linear sampling approaches to inverse scattering
}

\author{
Cédric Bellis ${ }^{\mathrm{a}, \mathrm{b}, *}$, Marc Bonnet ${ }^{\mathrm{c}}$, Bojan B. Guzina ${ }^{\mathrm{a}}$ \\ a Dept. of Civil Engineering, University of Minnesota, Minneapolis MN 55455, USA \\ b Solid Mechanics Laboratory (UMR CNRS 7649), École Polytechnique, Palaiseau, France \\ c POems (UMR 7231 CNRS-ENSTA-INRIA), ENSTA, Paris, France
}

\begin{abstract}
The focus of this study is the reconstruction of a penetrable obstacle in acoustic medium from the knowledge of incident time-harmonic waves and corresponding scattered fields. The problem is investigated by way of two competing approaches: the method of topological sensitivity and that of linear sampling, that have been successfully developed for a variety of physical settings (acoustic, electromagnetic, elastodynamic) as non-iterative tools for solving the inverse scattering problem. On adopting a particular scattering configuration - plane waves impigning on a spherical obstacle - that permits analytical treatment as the testing platform, a parallel is drawn between the two methods to evaluate their relative performance in reconstructing the obstacle from the scattered field data. For completeness, the comparison is made by considering a range of input parameters in terms of material properties of the scatterer, frequency of illuminating waves, and noise in the data.
\end{abstract}

Key words : Inverse scattering, topological sensitivity, linear sampling method, acoustic waves

\section{Introduction}

Inverse scattering of acoustic i.e. scalar waves by penetrable obstacles has been a keen area of research since the early twentieth century [25], continuing to draw attention to this day [21, 26, $33,38,39,43,51]$. Over the past two decades, the traditional linearization (e.g. weak scatterer) and non-linear minimization approaches $[8,21,46,49,50]$ to the problem have been accompanied by a disparate class of inverse scattering solutions, herein termed the "sampling" methods [45], that are both minimization-free and applicable to penetrable scatterers of arbitrary contrast. In particular these techniques, that are also referred to as qualitative methods [13], are capable of reasonably approximating the geometry of a generic (penetrable or impenetrable) scatterer with only a modest computational effort and no prior information about the nature of the obstacle. Such inverse scattering solutions commonly entail the development of an indicator function that, for a given sampling point, projects the observations of the scattered wavefield onto a suitable functional space synthesizing the wave motion in the reference (obstacle-free) domain. This indicator function, designed to reach extreme values when the sampling point strikes the scatterer, can be established from either mathematical or physical standpoint. Examples of the former paradigm for extended (i.e. arbirarily-sized) scatterers are the linear

\footnotetext{
${ }^{*}$ Current address: Dept. of Applied Physics and Applied Mathematics, Columbia University, New York NY 10027, USA
} 
sampling method $[11,13,18,19]$, the factorization method [32], the probe method [44, 45] and the weighted optimal control method [3], while among the sampling techniques for point-like scatterers one may mention the multiple signal classification (MUSIC) algorithm [4,47] and the direct method $[5,15]$. On the heuristic side of the coin, the sampling methods are perhaps best exemplified via the topological sensitivity approach $[14,24,27,35]$ that can be deemed as a counterpart of the direct method for extended scatterers.

From the foregoing array of non-iterative solutions, this study focuses on the methods of linear sampling and topological sensitivity. In the context of inverse scattering the latter technique, which quantifies the perturbation of a cost functional due to the nucleation of an infinitesimal defect in the reference (obstacle-free) domain, is used as an effective obstacle indicator through an assembly of sampling points where it attains extreme negative values. In contrast, the linear sampling method revolves around the solution of a particular integral equation, whose kernel is built from the observations of the scattered field, and whose solution is shown to be unbounded for sampling points (entering the right-hand side of the equation) outside of the support of the scatterer.

So far, unmediated appositions of the competing sampling methods have been relatively limited. They include a theoretical connection between the linear sampling method and MUSIC algorithm [16] for point-like scatterers; a numerical comparison between the linear sampling, factorization, and linear tomographic methods [12] for extended scatterers; a relationship between the factorization method and MUSIC algorithm for extended scatterers [7]; and, most recently, a comparison between the direct method, MUSIC algorithm, and Kirchhoff migration [2] for point-like scatterers. To the authors' knowledge, no commensurate studies exist on the relationship and comparative performance between the linear sampling and topological sensitivity methods - that are the focus of this work - pertaining to extended scatterers. To palliate the disparity in theoretical platforms of the two methods, the comparison is effected in a canonical setting that presupposes the "hidden" obstacle in the form of a penetrable ball that is concentric with the observation surface. Aided by such simplifying hypothesis, the competing indicator functions are computed explicitly in terms of series featuring spherical Bessel and Hankel functions. The analytical developments are illustrated via numerical results that highlight the performance of the two methods over a range input parameters such as the excitation frequency, material properties of the scatterer, and signal-to-noise ratio characterizing the data.

\section{Preliminaries}

Consider the scattering of acoustic waves by penetrable obstacle $B \subset \mathbb{R}^{3}$ in a homogeneous, lossless background medium endowed with mass density $\rho$ and elastic modulus $\kappa$. On denoting by $\rho_{\star}$ and $\kappa_{\star}$ the respective parameters inside the obstacle, the latter can be conveniently characterized by two dimensionless material constants, $\beta:=\rho / \rho_{\star}$ and $\eta:=\kappa / \kappa_{\star}$. Moreover, letting $c=\sqrt{\kappa / \rho}$ and $c_{\star}=\sqrt{\kappa_{\star} / \rho_{\star}}$ denote respectively the speed of sound in the background medium and the obstacle, the refraction index $\gamma=c / c_{\star}$ is introduced such that $\beta \gamma^{2}=\eta$. Given the entire set, $\Sigma$, of incident directions $\boldsymbol{\delta}$ (where $|\boldsymbol{\delta}|=1$ and $\Sigma$ is the unit sphere in $\mathbb{R}^{3}$ ), the obstacle is illuminated by time-harmonic plane waves $u_{\mathrm{i}}(\boldsymbol{\xi}, \boldsymbol{\delta}):=e^{\mathrm{i} k \boldsymbol{\xi} \cdot \boldsymbol{\delta}}$, where $k=\omega / c$ is the wavenumber and $\omega$ denotes the frequency of excitation. As usual, the temporal factor $e^{-i \omega t}$ is omitted for brevity. In this setting, the obstacle induces a perturbation of the incident field in the form of the scattered field, $u_{\mathrm{s}}$, for each direction of incidence $\boldsymbol{\delta}$. Accordingly, the total acoustic field $u$ in $\mathbb{R}^{3}$ can be decomposed as

$$
u(\boldsymbol{\xi}, \boldsymbol{\delta})=u_{\mathrm{s}}(\boldsymbol{\xi}, \boldsymbol{\delta})+u_{\mathrm{i}}(\boldsymbol{\xi}, \boldsymbol{\delta}) \quad\left(\boldsymbol{\xi} \in \mathbb{R}^{3} \backslash B, \boldsymbol{\delta} \in \Sigma\right) .
$$


For given $\boldsymbol{\delta}$, the forward scattering problem is governed by the field equations and boundary conditions

$$
\begin{array}{ll}
\Delta u_{\mathrm{s}}(\boldsymbol{\xi}, \boldsymbol{\delta})+k^{2} u_{\mathrm{s}}(\boldsymbol{\xi}, \boldsymbol{\delta})=0 & \left(\boldsymbol{\xi} \in \mathbb{R}^{3} \backslash \bar{B}\right) \\
\Delta u(\boldsymbol{\xi}, \boldsymbol{\delta})+\gamma^{2} k^{2} u(\boldsymbol{\xi}, \boldsymbol{\delta})=0 & (\boldsymbol{\xi} \in B) \\
u_{\mathrm{s}}(\boldsymbol{\xi}, \boldsymbol{\delta})+u_{\mathrm{i}}(\boldsymbol{\xi}, \boldsymbol{\delta})=u(\boldsymbol{\xi}, \boldsymbol{\delta}) & (\boldsymbol{\xi} \in \partial B) \\
u_{\mathrm{s}, \boldsymbol{n}}(\boldsymbol{\xi}, \boldsymbol{\delta})+u_{\mathrm{i}, \boldsymbol{n}}(\boldsymbol{\xi}, \boldsymbol{\delta})=\beta u_{, \boldsymbol{n}}(\boldsymbol{\xi}, \boldsymbol{\delta}) & (\boldsymbol{\xi} \in \partial B) \\
\lim _{|\xi| \rightarrow \infty} \boldsymbol{\nabla} u_{\mathrm{s}}(\boldsymbol{\xi}, \boldsymbol{\delta}) \cdot \boldsymbol{\xi}-\mathrm{i} k u_{\mathrm{s}}(\boldsymbol{\xi}, \boldsymbol{\delta})|\boldsymbol{\xi}|=0 &
\end{array}
$$

where $f_{, \boldsymbol{n}}(\boldsymbol{\xi})=\nabla f(\boldsymbol{\xi}) \cdot \boldsymbol{n}(\boldsymbol{\xi})$ denotes the derivative with respect to the unit outward normal $\boldsymbol{n}$ to $\partial B$, and $(2 \mathrm{e})$ signifies the Sommerfeld radiation condition.

To aid the ensuing discussion, it is useful to consider the limiting forms of $(2)$ as in $[23,30]$ by making reference to the velocity potential $\psi$ in $B$ such that

$$
u(\boldsymbol{\xi}, \boldsymbol{\delta})=\mathrm{i} \omega \rho_{*} \psi(\boldsymbol{\xi}, \boldsymbol{\delta})=\mathrm{i} \omega \frac{\rho}{\beta} \psi(\boldsymbol{\xi}, \boldsymbol{\delta}) \quad(\boldsymbol{\xi} \in B, \boldsymbol{\delta} \in \Omega) .
$$

When $\beta \rightarrow \infty$, one finds from (3) and hypothesis $|\psi|<\infty$ that $u \rightarrow 0$ in $B$, whereby (2) becomes the exterior Dirichlet problem for $u_{\mathrm{s}}$. In this case the obstacle is referred to as being sound-soft. When $\beta \rightarrow 0$, on the other hand, boundary condition $(2 \mathrm{~d})$ becomes homogeneous and (2) reduces to the exterior Neumann problem for $u_{\mathrm{s}}$. In this situation, the obstacle is referred to as being sound-hard.

\subsection{Inverse problem}

The inverse problem at hand consists of reconstructing the support $B$ and material properties $(\beta, \eta)$ of a hidden penetrable obstacle from the measurements of the scattered field $u_{\text {obs }}(\boldsymbol{\xi}, \boldsymbol{\delta})$ over an observation surface $S_{\text {obs }}$, induced by the set of incident plane waves $u_{\mathrm{i}}(\boldsymbol{\xi}, \boldsymbol{\delta}), \boldsymbol{\delta} \in \Sigma$. In what follows, two non-iterative reconstruction techniques, rooted respectively in the concepts of topological sensitivity and linear sampling, will be investigated analytically and numerically for a canonical scattering configuration.

\subsubsection{Method of topological sensitivity}

This approach assumes the inverse problem to be formulated in terms of the minimization of a cost functional $\mathcal{J}\left(B^{\prime}, \beta^{\prime}, \eta^{\prime}\right)$ quantifying the misfit between the true obstacle $(B, \beta, \eta)$ and its trial companion $\left(B^{\prime}, \beta^{\prime}, \eta^{\prime}\right)$, of the form

$$
\mathcal{J}\left(B^{\prime}, \beta^{\prime}, \eta^{\prime}\right)=\int_{\Sigma} \int_{S_{\mathrm{obs}}} \varphi\left(u_{\mathrm{s}}\left[B^{\prime}, \beta^{\prime}, \eta^{\prime}\right], \boldsymbol{\xi}, \boldsymbol{\delta}\right) \mathrm{d} \boldsymbol{\xi} \mathrm{d} \boldsymbol{\delta},
$$

where $u_{\mathrm{s}}^{\prime}=u_{\mathrm{s}}\left[B^{\prime}, \beta^{\prime}, \eta^{\prime}\right]$ denotes the scattered field solving $(2)$ with $(B, \beta, \eta)$ superseded by $\left(B^{\prime}, \beta^{\prime}, \eta^{\prime}\right)$. The misfit density $\varphi$ signifies a measure of distance between $u_{\mathrm{s}}^{\prime}$ and $u_{\mathrm{obs}}$, and is hereon assumed to take the customary least-squares format

$$
\varphi\left(u_{\mathrm{s}}^{\prime}, \boldsymbol{\xi}, \boldsymbol{\delta}\right)=\frac{1}{2}\left|u_{\mathrm{s}}^{\prime}(\boldsymbol{\xi}, \boldsymbol{\delta})-u_{\mathrm{obs}}(\boldsymbol{\xi}, \boldsymbol{\delta})\right|^{2} \quad\left(\boldsymbol{\xi} \in S_{\mathrm{obs}}, \boldsymbol{\delta} \in \Sigma\right) .
$$

Designed to circumvent computationally-intensive minimization of $\mathcal{J}$, the method of topological sensitivity (MTS) investigates the behavior of $\mathcal{J}$ for trial defects $B^{\prime}=B_{\mathbf{x}, \varepsilon}:=\mathbf{x}+\varepsilon \mathcal{B}$ of vanishing size $\varepsilon$ at "sampling" locations $\mathbf{x} \in \mathbb{R}^{3}$, where the fixed bounded set $\mathcal{B} \subset \mathbb{R}^{3}$ contains the origin and specifies the shape of $B_{\mathbf{x}, \varepsilon}$. In this approach, the vanishing obstacle is endowed 
with trial material properties $\beta^{\prime}$ and $\eta^{\prime}$ that do not necessarily coincide with their "true" counterparts $\beta$ and $\eta$. Following e.g. $[27,48]$, one seeks the asymptotic behavior of $\mathcal{J}\left(B_{\mathbf{x}, \varepsilon}, \beta^{\prime}, \eta^{\prime}\right)$ as $\varepsilon \rightarrow 0$ through an expansion of the form

$$
\mathcal{J}\left(B_{\mathbf{x}, \varepsilon}, \beta^{\prime}, \eta^{\prime}\right) \underset{\varepsilon \rightarrow 0}{=} \mathcal{J}\left(\emptyset, \beta^{\prime}, \eta^{\prime}\right)+\eta(\varepsilon)|\mathcal{B}| \mathcal{T}\left(\mathbf{x}, \beta^{\prime}, \eta^{\prime}\right)+o(\eta(\varepsilon)),
$$

where $\mathcal{J}\left(\emptyset, \beta^{\prime}, \eta^{\prime}\right)$ denotes the cost functional evaluated without obstacle; $|\mathcal{B}|$ is the volume of $\mathcal{B}$, and $\eta(\varepsilon)>0$ is a monotonic homogeneous function of $\varepsilon>0$. Provided that the expansion of form (6) exists (as is known to be the case for the present problem, see [27]), coefficient $\mathcal{T}\left(\mathbf{x}, \beta^{\prime}, \eta^{\prime}\right)$ is called the topological sensitivity of $\mathcal{J}$ at $\mathbf{x} \in \mathbb{R}^{3}$. In this setting, the sampling points $\mathbf{x}$ at which $\mathcal{T}\left(\mathbf{x}, \beta^{\prime}, \eta^{\prime}\right)$ attains pronounced negative values are deemed to belong to the support of $B$, as $\mathcal{J}\left(B_{\mathbf{x}, \varepsilon}, \beta^{\prime}, \eta^{\prime}\right)$ then decreases with $\varepsilon$ for sufficiently small $\varepsilon$. The use of $\mathcal{T}\left(\mathbf{x}, \beta^{\prime}, \eta^{\prime}\right)$ as a defect indicator function accordingly consists in determining the spatial region $B_{\mathrm{MTS}}(\alpha)$ such that

$$
B_{\mathrm{MTS}}(\alpha)=\left\{\mathbf{x} \in \mathbb{R}^{3}: \mathcal{T}\left(\mathbf{x}, \beta^{\prime}, \eta^{\prime}\right) \leqslant \alpha \min _{\boldsymbol{\xi} \in \mathbb{R}^{3}} \mathcal{T}\left(\boldsymbol{\xi}, \beta^{\prime}, \eta^{\prime}\right)<0\right\}
$$

for given threshold $\alpha \in[0,1]$. While largely heuristic at this point, this approach is wellsupported by numerical experiments $[10,27,28,36]$. Moreover, as shown in $[27,28]$, a search for the optimal values of $\beta^{\prime}$ and $\eta^{\prime}$ that minimize $\mathcal{T}\left(\mathbf{x}, \beta^{\prime}, \eta^{\prime}\right)<0$ (with $\mathbf{x}$ fixed) provides the basis for qualitative material identification of $B$.

For the problem under consideration, the topological sensitivity $\mathcal{T}$ and asymptotic scaling function $\eta(\varepsilon)$ are shown to be given by

$$
\mathcal{T}\left(\mathbf{x}, \beta^{\prime}, \eta^{\prime}\right)=\int_{\Sigma} \operatorname{Re}\left[\left(1-\beta^{\prime}\right) \nabla u_{\mathrm{a}} \cdot \mathcal{A} \cdot \nabla u_{\mathrm{i}}-\left(1-\eta^{\prime}\right) k^{2} u_{\mathrm{a}} u_{\mathrm{i}}\right](\mathbf{x}, \boldsymbol{\delta}) \mathrm{d} \boldsymbol{\delta}, \quad \eta(\varepsilon)=\varepsilon^{3},
$$

see [27]. Here $u_{\mathrm{i}}$ is the incident field defined earlier; $\mathcal{A}=\mathcal{A}\left(\mathcal{B}, \beta^{\prime}\right)$ is the so-called polarization tensor synthesizing the shape and material properties of the vanishing $\operatorname{defect}[6,27] ; u_{\mathrm{a}}(\cdot, \boldsymbol{\delta})$ denotes the adjoint field, given by the single-layer potential

$$
u_{\mathrm{a}}(\boldsymbol{\xi}, \boldsymbol{\delta})=\int_{S_{\mathrm{obs}}} \partial_{u} \varphi(0, \boldsymbol{\zeta}, \boldsymbol{\delta}) G(\boldsymbol{\xi}, \boldsymbol{\zeta}) \mathrm{d} \boldsymbol{\zeta} \quad\left(\boldsymbol{\xi} \in \mathbb{R}^{3}, \boldsymbol{\delta} \in \Sigma\right)
$$

where

$$
G(\boldsymbol{\xi}, \mathbf{x}):=\frac{e^{\mathrm{i} k|\boldsymbol{\xi}-\mathbf{x}|}}{4 \pi|\boldsymbol{\xi}-\mathbf{x}|}
$$

is the fundamental solution of the Helmholtz equation, and the partial derivative $\partial_{u} \varphi$ of the (real-valued) misfit density function $\varphi$ is defined in terms of the real and imaginary parts of $u$ as

$$
\partial_{u} \varphi:=\partial_{\operatorname{Re}(u)} \varphi-\mathrm{i} \partial_{\operatorname{Im}(u)} \varphi
$$

Assuming the least-squares cost functional (5) and noise-free data (i.e. $u_{\mathrm{obs}}=u_{\mathrm{s}}$ on $S_{\mathrm{obs}}$ ), (11) reduces to

$$
\partial_{u} \varphi=-\bar{u}_{\mathrm{s}}(\boldsymbol{\xi}, \boldsymbol{\delta}) .
$$

where overbar denotes complex conjugation. In the case of an infinitesimal spherical obstacle, for which $\mathcal{B}$ is the unit ball, one has

$$
|\mathcal{B}|=\frac{4 \pi}{3}, \quad \mathcal{A}\left(\mathcal{B}, \beta^{\prime}\right)=\frac{3}{2+\beta^{\prime}} \mathcal{I}_{2}
$$

where $\mathcal{I}_{2}$ is the second-order identity tensor, see [27]. In this article, the MTS will be considered only for spherical trial inclusions, for which (13) holds. To emphasize and better distinguish 
the dependence of $\mathcal{T}$ on the material parameters of a trial inclusion $\left(\beta^{\prime}, \eta^{\prime}\right)$ and those of the "true" obstacle $(\beta, \eta)$, it is convenient to employ the decomposition

$$
\mathcal{T}\left(\mathbf{x}, \beta^{\prime}, \eta^{\prime}\right)=\frac{3\left(1-\beta^{\prime}\right)}{\left(2+\beta^{\prime}\right)} \mathcal{T}^{\beta}(\mathbf{x}, \beta, \eta)+\left(1-\eta^{\prime}\right) \mathcal{T}^{\eta}(\mathbf{x}, \beta, \eta)
$$

where the "dipole" term $\mathcal{T}^{\beta}$ and "monopole" term $\mathcal{T}^{\eta}$ correspond to trial obstacles featuring exclusively the contrast in mass density and bulk modulus, respectively.

Here it is worth noting that (i) both the incident and adjoint fields entering (8) are defined for the obstacle-free background medium $\mathbb{R}^{3}$, and (ii) the adjoint solution carries the experimental information about hidden obstacle $(B, \beta, \eta)$ via misfit density $\partial_{u} \varphi(0, \cdot, \cdot)$ in $(9)$.

\subsubsection{Linear sampling method}

The linear sampling method (LSM) $[13,31]$ had been originally proposed for inverse acoustic scattering problems based on far-field data [19], and later extended to near-field observations $[18,41]$. In what follows, the discussion conjugates the two approaches. Letting the near-field operator $\mathcal{S}$ be defined by

$$
\mathcal{S}: L^{2}(\Sigma) \rightarrow L^{2}\left(S_{\mathrm{obs}}\right), \quad[\mathcal{S} f](\boldsymbol{\xi}):=\int_{\Sigma} u_{\mathrm{obs}}(\boldsymbol{\xi}, \boldsymbol{\delta}) f(\boldsymbol{\delta}) \mathrm{d} \boldsymbol{\delta},
$$

the linear sampling method consists in seeking, for a given sampling point $\mathbf{x} \in \mathbb{R}^{3}$, a function $g(\mathbf{x}, \cdot) \in L^{2}(\Sigma)$ that solves the linear integral equation

$$
[\mathcal{S} g(\mathbf{x}, \cdot)](\boldsymbol{\xi})=G(\boldsymbol{\xi}, \mathbf{x}) \quad\left(\boldsymbol{\xi} \in S_{\text {obs }}\right) .
$$

It is important to emphasize that, since the operator $\mathcal{S}$ is compact from $L^{2}(\Sigma)$ into $L^{2}\left(S_{\text {obs }}\right)$, the equation (16) is ill-posed [34]. Nevertheless, the resolution of the integral equation (16) is based on the result [20] that $\mathcal{S}$ is injective with dense range if and only if the so-called homogeneous interior transmission problem

$$
\begin{array}{lll}
\text { (a) } \quad \Delta v(\boldsymbol{\xi})+k^{2} v(\boldsymbol{\xi})=0, & \text { (b) } \Delta w(\boldsymbol{\xi})+\gamma^{2} k^{2} w(\boldsymbol{\xi})=0 & (\boldsymbol{\xi} \in B) \\
\text { (c) } v(\boldsymbol{\xi})=w(\boldsymbol{\xi}), & \text { (d) } v_{, \boldsymbol{n}}(\boldsymbol{\xi})=\beta w_{, \boldsymbol{n}}(\boldsymbol{\xi}) & (\boldsymbol{\xi} \in \partial B)
\end{array}
$$

does not admit a pair of solutions $(v, w)$ such that $v$ has the form

$$
v(\boldsymbol{\xi})=\int_{\Sigma} u_{\mathrm{i}}(\boldsymbol{\xi}, \boldsymbol{\delta}) f(\boldsymbol{\delta}) \mathrm{d} \boldsymbol{\delta} .
$$

with some non-zero density $f \in L^{2}(\Sigma)$ (note that such $v$ is known as a Herglotz wave function with density $f$ ).

For values of $k$ such that problem (17) has a non-trivial solution, known as transmission eigenvalues, the linear sampling method fails, lending great importance to the study of their properties [31,42]. Otherwise, it can be shown [13] that, while (16) is in general not exactly solvable, for every $\varepsilon>0$ there exists an approximate solution $g_{\varepsilon}(\mathbf{x}, \cdot) \in L^{2}(\Sigma)$ such that

$$
\left\|\mathcal{S} g_{\varepsilon}(\mathbf{x}, \cdot)-G(\cdot, \mathbf{x})\right\|_{L^{2}\left(S_{\mathrm{obs}}\right)}<\varepsilon .
$$

The linear sampling method is supported by the key properties that: (i) if $\mathbf{x} \in B$, then $\left\|g_{\varepsilon}(\mathbf{x}, \cdot)\right\|_{L^{2}(\Sigma)} \rightarrow \infty$ as $\mathbf{x} \rightarrow \mathbf{y} \in \partial B$, and (ii) if $\mathbf{x} \in \mathbb{R}^{3} \backslash B$, then $\left\|g_{\varepsilon}(\mathbf{x}, \cdot)\right\|_{L^{2}(\Sigma)} \rightarrow \infty$ as $\varepsilon \rightarrow 0$ (see e.g. $[13,18]$, and also $[29,41]$ for elasticity). In other words, $g_{\varepsilon}(\mathbf{x}, \cdot)$ is (almost) unbounded for sampling points $\mathbf{x}$ outside $B$; moreover $g_{\varepsilon}(\mathbf{x}, \cdot)$ blows up as $\mathbf{x}$ approaches $\partial B$ from within $B$. 
Due to the compactness of $\mathcal{S}$, a numerical solution of (16) necessitates the use of suitable regularization techniques [13] such as Tikhonov regularization. Considering the effects of regularization and imperfect observations, one expects to have

$$
\left\|g_{\varepsilon}\left(\mathbf{x}_{e}, \cdot\right)\right\|_{L^{2}(\Sigma)} \gg\left\|g_{\varepsilon}\left(\mathbf{x}_{i}, \cdot\right)\right\|_{L^{2}(\Sigma)} \quad\left(\mathbf{x}_{e} \in \mathbb{R}^{3} \backslash B, \mathbf{x}_{i} \in B\right)
$$

in a practical situation. Accordingly, the support of $B$ can be reconstructed as $B_{\mathrm{LSM}}:=$ $\mathbb{R}^{3} \backslash\left\{\mathbf{x} \in \mathbb{R}^{3}, I_{\mathrm{LSM}}(\mathbf{x}) \ll 1\right\}$ via the use of the indicator function

$$
I_{\mathrm{LSM}}(\mathbf{x}):=\left(\left\|g_{\varepsilon}(\mathbf{x}, \cdot)\right\|_{L^{2}(\Sigma)}\right)^{-1} \text {. }
$$

In the ensuing LSM developments, the subscript " $\varepsilon$ " is dropped for simplicity of notation.

\subsection{Penetrable spherical obstacle}

This study focuses on the canonic case when the scatterer $B$ is a penetrable ball in $\mathbb{R}^{3}$. To facilitate the analytical developments, the radius of $B$ is taken as unity, whereby the wavenumber $k$ and all other length scales are to be understood as dimensionless (i.e. normalized by the radius of the ball). The set of incident plane-wave directions deployed to illuminate the ball is the unit sphere $\Sigma$, while the observation surface $S_{\text {obs }}$ is taken as sphere of radius $R$ (i.e. $S_{\text {obs }}=R \Sigma$ ) that is concentric with $B$. With such premises, the forward and adjoint problems have analytical solutions, given next, from which the analytical formulations of the MTS and the LSM are derived and investigated thereon.

Forward solution. Both the MTS and the LSM exploit observations of the scattered field $u_{\mathrm{s}}$ over $S_{\mathrm{obs}}$, as induced by $u_{\mathrm{i}}$ acting on $(B, \beta, \eta)$. For the assumed canonic configuration, the scalar fields $u_{\mathrm{s}}$ and $u$ featured in the forward problem (2) are respectively defined in $\mathbb{R}^{3} \backslash B$ and in $B$, and admit expansions of the form

$$
\begin{aligned}
& u_{\mathrm{s}}(\boldsymbol{\xi}, \boldsymbol{\delta})=\sum_{\ell=0}^{+\infty} \sum_{m=-\ell}^{+\ell} \lambda_{\ell}^{m}(\boldsymbol{\delta}) h_{\ell}(k|\boldsymbol{\xi}|) Y_{\ell}^{m}(\hat{\boldsymbol{\xi}}) \quad\left(\boldsymbol{\xi} \in \mathbb{R}^{3} \backslash B, \boldsymbol{\delta} \in \Sigma\right), \\
& u(\boldsymbol{\xi}, \boldsymbol{\delta})=\sum_{\ell=0}^{+\infty} \sum_{m=-\ell}^{+\ell} \mu_{\ell}^{m}(\boldsymbol{\delta}) j_{\ell}(\gamma k|\boldsymbol{\xi}|) Y_{\ell}^{m}(\hat{\boldsymbol{\xi}}) \quad(\boldsymbol{\xi} \in B, \boldsymbol{\delta} \in \Sigma),
\end{aligned}
$$

see [40], where $j_{\ell}$ and $h_{\ell}$ are the spherical Bessel and Hankel functions of the first kind and order $\ell,\left(Y_{\ell}^{m}\right)_{\ell \in \mathbb{N},-\ell \leqslant m \leqslant+\ell}$ are the orthonormal spherical harmonics, and $\hat{\boldsymbol{\xi}}:=\boldsymbol{\xi} /|\boldsymbol{\xi}|$ is the unit position vector. The expansion coefficients $\lambda_{\ell}^{m}(\boldsymbol{\delta}), \mu_{l}^{m}(\boldsymbol{\delta})$ depend on the direction of incidence $\boldsymbol{\delta}$ and are found by employing the transmission conditions over $\partial B$, the Jacobi-Anger expansion (66) and the orthonormality of spherical harmonics (62). As a result, the scattered field $u_{\mathrm{s}}$ is given by

$$
u_{\mathrm{s}}(\boldsymbol{\xi}, \boldsymbol{\delta})=\sum_{\ell=0}^{+\infty} \mathrm{i}^{\ell}(2 \ell+1) \Lambda_{\ell}(\beta, \eta ; k) h_{\ell}(k|\boldsymbol{\xi}|) P_{\ell}(\hat{\boldsymbol{\xi}} \cdot \boldsymbol{\delta})
$$

where $P_{\ell}$ denotes the Legendre polynomial of order $\ell$, and

$$
\Lambda_{\ell}(\beta, \eta ; k)=-\frac{j_{\ell}^{\prime}(k)-\alpha_{\ell}(\beta, \eta) j_{\ell}(k)}{h_{\ell}^{\prime}(k)-\alpha_{\ell}(\beta, \eta) h_{\ell}(k)}, \quad \alpha_{\ell}(\beta, \eta)=\sqrt{\beta \eta} \frac{j_{\ell}^{\prime}(k \sqrt{\eta / \beta})}{j_{\ell}(k \sqrt{\eta / \beta})} .
$$

Note that $j_{\ell}^{\prime}, h_{\ell}^{\prime}$ denote the derivatives of $j_{\ell}, h_{\ell}$ with respect to their argument, and that each $u_{\mathrm{s}}(\cdot, \boldsymbol{\delta})$ is axisymmetric around direction $\boldsymbol{\delta}$. Moreover, the limiting cases $\sqrt{\beta \eta} \rightarrow 0$ and $\sqrt{\beta \eta} \rightarrow$ $+\infty$ respectively yield $\Lambda_{\ell}(\beta, \eta ; k)=-j_{\ell}^{\prime}(k) / h_{\ell}^{\prime}(k)$ (corresponding to the sound-hard obstacle, $\partial_{n}\left(u_{\mathrm{s}}+u_{\mathrm{i}}\right)=0$ on $\left.\partial B\right)$ and $\Lambda_{\ell}(\beta, \eta ; k)=-j_{\ell}(k) / h_{\ell}(k)$ (corresponding to the sound-soft obstacle, $u_{\mathrm{s}}+u_{\mathrm{i}}=0$ on $\left.\partial B\right)$. 
Adjoint solution. To facilitate the computation of topological sensitivity (8), it is useful to evaluate the adjoint field given by (9) and (12) analytically. By way of (23), series representation (68) of the fundamental solution $G(\boldsymbol{\xi}, \boldsymbol{\zeta})$ (the Gegenbauer theorem), the addition theorem (63), and the orthonormality property (62), the adjoint field is found to be given by

$$
u_{\mathrm{a}}(\boldsymbol{\xi}, \boldsymbol{\delta})=-k R^{2} \sum_{\ell=0}^{+\infty}(-1)^{\ell} \mathrm{i}^{\ell+1}(2 \ell+1) \bar{\Lambda}_{\ell}(\beta, \eta ; k)\left|h_{\ell}(k R)\right|^{2} j_{\ell}(k|\boldsymbol{\xi}|) P_{\ell}(\hat{\boldsymbol{\xi}} \cdot \boldsymbol{\delta}) \quad(|\boldsymbol{\xi}| \leq R, \boldsymbol{\delta} \in \Sigma) .
$$

This expression relies on the Gegenbauer theorem (68), and hence it applies only inside a closed ball $B_{R}$ of radius $R$ bounded by $S_{\text {obs }}=R \Sigma$. This is consistent with the implicit assumption that the measurement surface $S_{\text {obs }}$ surrounds the hidden obstacle, so that $\mathcal{T}$ needs only be evaluated inside $B_{R}$.

\section{Method of topological sensitivity: spherical obstacle}

Analytical expression. On substituting the adjoint solution (25) and the Jacobi-Anger expansion (66) of $u_{\mathrm{i}}$ into (6) with $\mathcal{A}$ given by (13), invoking properties (62), (64) and (65), and performing the integration over $\boldsymbol{\delta} \in \Sigma$, the dipole and monopole components of the topological derivative are found to be given respectively by

$$
\mathcal{T}^{\star}(\mathbf{x}, \beta, \eta)=\sum_{\ell=0}^{+\infty} \mathcal{T}_{\ell}^{\star}(\mathbf{x}, \beta, \eta) \quad(\star=\beta, \eta),
$$

where

$$
\mathcal{T}_{\ell}^{\beta}(\mathbf{x}, \beta, \eta)=-Q_{\ell} R_{\ell}^{\beta}(\mathbf{x}) \operatorname{Re}\left[\mathrm{i} \bar{\Lambda}_{\ell}(\beta, \eta ; k)\right], \quad \mathcal{T}_{\ell}^{\eta}(\mathbf{x}, \beta, \eta)=-Q_{\ell} R_{\ell}^{\eta}(\mathbf{x}) \operatorname{Re}\left[\mathrm{i} \bar{\Lambda}_{\ell}(\beta, \eta ; k)\right],
$$

$\Lambda_{\ell}(\beta, \eta ; k)$ is given by $(24)$, and

$$
\begin{aligned}
R_{\ell}^{\beta}(\mathbf{x}) & :=\ell(\ell+1) j_{\ell}(k|\mathbf{x}|)^{2}+k^{2} j_{\ell}^{\prime}(k|\mathbf{x}|)^{2}, \quad R_{\ell}^{\eta}(\mathbf{x}):=-k^{2} j_{\ell}(k|\mathbf{x}|)^{2}, \\
Q_{\ell} & :=4 \pi k R^{2}(2 \ell+1)\left|h_{\ell}(k R)\right|^{2} .
\end{aligned}
$$

Note that $\mathcal{T}_{\ell}^{\beta}$ and $\mathcal{T}_{\ell}^{\eta}$ depend on the true physical constants of a hidden obstacle, while their multipliers of according to (8) and (26) depend on the trial counterparts of a vanishing inclusion.

Series truncation. Clearly, relation (26) involves infinite summation over index $\ell$ which has to be truncated for computational purposes. On employing the large-order asymptotic formulae from Appendix B, one has

$$
\begin{gathered}
Q_{\ell}=\frac{16 \pi \mathrm{i}}{\ell \rightarrow \infty}\left(\frac{2 \ell}{e k R}\right)^{2 \ell}\left(1+O\left(\ell^{-1}\right)\right), \\
\Lambda_{\ell}(\beta, \eta ; k) \underset{\substack{\ell \rightarrow \infty \\
\ell \rightarrow \infty}}{=} \frac{\mathrm{i} k}{4 \ell} \frac{-\beta}{1+\beta}\left(\frac{e k}{2 \ell}\right)^{2 \ell}\left(1+O\left(\ell^{-1}\right)\right), \\
R_{\ell}^{\beta}(\mathbf{x}) \underset{\substack{\ell \rightarrow \infty \\
\ell \rightarrow \infty}}{=}\left[1+\frac{k^{2}}{|\mathbf{x}|^{2}}\right]\left(\frac{e k|\mathbf{x}|}{2 \ell}\right)^{2 \ell}\left(1+O\left(\ell^{-1}\right)\right), \\
R_{\ell}^{\eta}(\mathbf{x}) \underset{\substack{\ell \rightarrow \infty \\
\ell \rightarrow \infty}}{=}-\frac{1}{8 \ell^{2}}\left(\frac{e k|\mathbf{x}|}{2 \ell}\right)^{2 \ell}\left(1+O\left(\ell^{-1}\right)\right) .
\end{gathered}
$$


where $e$ denotes Euler's number, whereby the large-order asymptotic behavior of $\mathcal{T}_{\ell}^{\beta}$ and $\mathcal{T}_{\ell}^{\eta}$ is such that

$$
\mathcal{T}_{\ell}^{\star}(\mathbf{x}, \beta, \eta) \underset{\ell \rightarrow \infty}{=} O\left(\left(\frac{e k|\mathbf{x}|}{2 R \ell}\right)^{2 \ell}\right) \quad(\star=\beta, \eta)
$$

Hence, $\mathcal{T}_{\ell}(\mathbf{x}, \beta, \eta)$ decays at a faster-than-exponential rate as $\ell \rightarrow \infty$, which indicates that the series (26) may, for numerical evaluation purposes, be safely truncated at some level $\ell_{0}$. Note, however, that the decay of $\mathcal{T}_{\ell}$ starts only for values of $\ell$ beyond the threshold defined by $e k|\mathbf{x}| /(2 R \ell)=1$ whose value increases with $k$, so the truncation level $\ell_{0}$ must be adjusted accordingly (see Fig. 1).

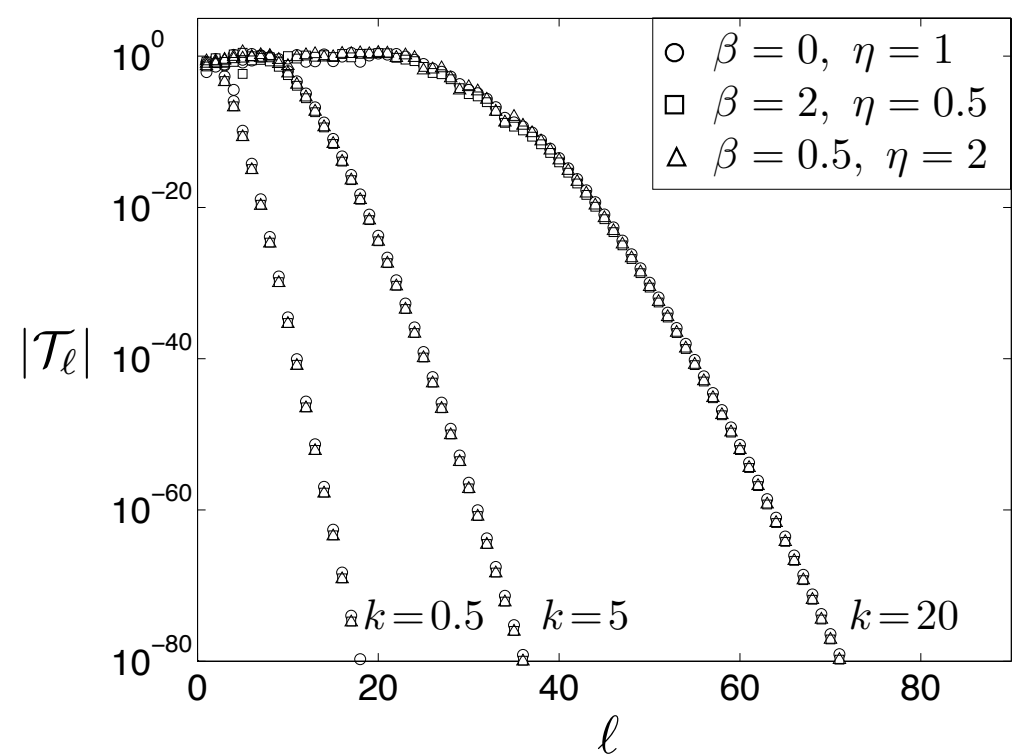

Figure 1: $\mathcal{T}_{\ell}(\mathbf{x}, \beta, \eta)$ versus the expansion index $\ell$ for sample values of the wavenumber $k$ and material parameters $(\beta, \eta)$.

\subsection{Behavior of topological sensitivity in limiting situations}

Asymptotic behavior for remote sampling points. The large-argument asymptotic properties (72) of $j_{\ell}$ and (76) of $j_{\ell}^{\prime}$ imply that $\left|\mathcal{T}\left(\mathbf{x}, \beta^{\prime}, \eta^{\prime}\right)\right|$ decays as $|\mathbf{x}| \rightarrow \infty$; more precisely:

$$
\left|\mathcal{T}\left(\mathbf{x}, \beta^{\prime}, \eta^{\prime}\right)\right|=O\left(|\mathbf{x}|^{-2}\right)
$$

This implies (as later shown on numerical results) that $\mathcal{T}\left(\mathbf{x}, \beta^{\prime}, \eta^{\prime}\right)$ does not (falsely) indicate non-existent obstacles at remote locations.

Low-frequency behavior. On employing small-argument asymptotic formulae (71) and (75) in (26), one has

$$
\begin{gathered}
Q_{\ell} \underset{k \rightarrow 0}{=} 4 \pi \frac{(2 \ell-1) ! !(2 \ell+1) ! !}{k(k R)^{2 \ell}}(1+O(k)), \\
\Lambda_{\ell}(\beta, \eta ; k) \underset{k \rightarrow 0}{=} \frac{1}{\mathrm{i}} \frac{1-\beta}{1+\beta+\ell^{-1}} \frac{k^{2 \ell+1}}{(2 \ell-1) ! !(2 \ell+1) ! !}(1+O(k)), \\
R_{\ell}^{\beta}(\mathbf{x}) \underset{k \rightarrow 0}{=} \frac{(k \ell)^{2}(k|\mathbf{x}|)^{2 \ell-2}}{(2 \ell+1) !^{2}}\left(1+\left(\ell^{-1}+1\right)|\mathbf{x}|^{2}\right)(1+O(k)), \\
R_{\ell}^{\eta}(\mathbf{x}) \underset{k \rightarrow 0}{=}-\frac{k^{2}(k|\mathbf{x}|)^{2 \ell}}{(2 \ell+1) ! !^{2}}(1+O(k)) .
\end{gathered}
$$


(for $\ell>0$ ), so that the low-frequency asymptotic behavior of $\mathcal{T}_{\ell}^{\beta}$ and $\mathcal{T}_{\ell}^{\eta}$ reads

$$
\begin{aligned}
\mathcal{T}_{\ell}^{\beta}(\mathbf{x}, \beta, \eta) & =\frac{4 \pi(1-\beta)}{1+\beta+\ell^{-1}} \frac{(k \ell)^{2}(k|\mathbf{x}|)^{2 \ell-2}}{(2 \ell+1) !^{2} R^{2 \ell}}\left(1+\left(\ell^{-1}+1\right)|\mathbf{x}|^{2}\right)(1+O(k)) \\
\mathcal{T}_{\ell}(\mathbf{x}, \beta, \eta) & =-\frac{4 \pi(1-\beta)}{1+\beta+\ell^{-1}} \frac{k^{2}(k|\mathbf{x}|)^{2 \ell}}{(2 \ell+1) ! !^{2} R^{2 \ell}}(1+O(k))
\end{aligned}
$$

The case $\ell=0$ must be treated separately, using (71) and (77). One obtains

$$
Q_{0}=-\frac{4 \pi}{k}, \quad \Lambda_{0}(\beta, \eta ; k)=-\frac{\mathrm{i} k^{3}}{3}(1-\eta), \quad R_{\ell}^{\beta}(\mathbf{x})=\frac{1}{9} k^{4}|\mathbf{x}|^{2}, \quad R_{\ell}^{\eta}(\mathbf{x})=-k^{2}
$$

(ignoring contributions of higher-order in $k$ ), and hence

$$
\mathcal{T}_{0}^{\beta}(\mathbf{x}, \beta, \eta)=\frac{4 \pi k^{6}}{27}(1-\eta)|\mathbf{x}|^{2}(1+O(k)), \quad \mathcal{T}_{0}^{\eta}(\mathbf{x}, \beta, \eta)=-\frac{4 \pi k^{4}}{3}(1-\eta)(1+O(k))
$$

Retaining only the leading (i.e. lowest-order) contributions in (32) and (33), the asymptotic behavior of $\mathcal{T}_{\ell}^{\beta}$ and $\mathcal{T}_{\ell}^{\eta}$ in the low-frequency limit is finally obtained as

$$
\begin{aligned}
& \mathcal{T}^{\beta}(\mathbf{x}, \beta, \eta)=\frac{4 \pi(1-\beta)}{2+\beta} \frac{k^{2}}{9 R^{2}}\left(1+2|\mathbf{x}|^{2}\right)+o\left(k^{2}\right) \\
& \mathcal{T}^{\eta}(\mathbf{x}, \beta, \eta)=-\left\{\frac{4 \pi}{3}(1-\eta)+\frac{4 \pi(1-\beta)}{2+\beta} \frac{|\mathbf{x}|^{2}}{9 R^{2}}\right\} k^{4}+o\left(k^{4}\right)
\end{aligned}
$$

Weak scatterer approximation. Consider the case of a weak scatterer, for which the physical contrasts that characterize the true obstacle are small, i.e. $|\beta-1| \ll 1$ and $|\eta-1| \ll 1$. In this case, the topological sensitivity (14) depends on the latter only through coefficients $\Lambda_{\ell}(\beta, \eta ; k)$, given by $(24)$. Letting $\beta=1+\hat{\beta}$ and $\eta=1+\hat{\eta}$ and noting that $(\mathrm{i}) \Lambda_{\ell}(1,1 ; k)=0$ and (ii) $(\beta, \eta) \mapsto \Lambda_{\ell}(\beta, \eta ; k)$ is differentiable with respect to $\beta, \eta$ at $(\beta, \eta)=(1,1)$, the first-order Taylor expansion of $\Lambda_{\ell}$ about $(\beta, \eta)=(1,1)$ takes the form

$$
\operatorname{Re}\left[\mathrm{i} \bar{\Lambda}_{\ell}(\beta, \eta ; k)\right]=\Lambda_{\ell}^{\beta}(k) \hat{\beta}+\Lambda_{\ell}^{\eta}(k) \hat{\eta}+o(|\hat{\beta}|+|\hat{\eta}|)
$$

where $\Lambda_{\ell}^{\beta}, \Lambda_{\ell}^{\eta}$ are found, after exploiting the properties of spherical Bessel functions, to be given by

$$
\begin{aligned}
& \Lambda_{\ell}^{\beta}(k):=\partial_{\beta} \operatorname{Re}\left[\mathrm{i} \bar{\Lambda}_{\ell}(\beta, \eta ; k)\right](1,1 ; k)=-\frac{k}{2}\left[3 k j_{\ell}(k) j_{\ell}^{\prime}(k)+k^{2} j_{\ell}^{\prime 2}(k)+k^{2} j_{\ell}^{2}(k)-\ell(\ell+1)\right] \\
& \Lambda_{\ell}^{\eta}(k):=\partial_{\eta} \operatorname{Re}\left[\mathrm{i} \bar{\Lambda}_{\ell}(\beta, \eta ; k)\right](1,1 ; k)=\frac{k}{2}\left[k j_{\ell}(k) j_{\ell}^{\prime}(k)+k^{2} j_{\ell}^{\prime 2}(k)+k^{2} j_{\ell}^{2}(k)-\ell(\ell+1)\right]
\end{aligned}
$$

On substituting expansions (35) into (26), one finds by way of (14) and (27) the topological sensitivity to read $\mathcal{T}=\sum_{\ell \geq 0} \mathcal{T}_{\ell}$ where $\mathcal{T}_{\ell}$ is given, to the first order in $\hat{\beta}$ and $\hat{\eta}$, by

$$
\mathcal{T}_{\ell}(\mathbf{x}, \beta, \eta)=Q_{\ell}\left[\Lambda_{\ell}^{\beta}(k) \hat{\beta}+\Lambda_{\ell}^{\eta}(k) \hat{\eta}\right]\left\{R_{\ell}^{\beta}(\mathbf{x}) \frac{3 \hat{\beta}^{\prime}}{3+\hat{\beta}^{\prime}}+R_{\ell}^{\eta}(\mathbf{x}) \hat{\eta}^{\prime}\right\}+o(|\hat{\beta}|+|\hat{\eta}|) .
$$

Since $Q_{\ell}>0, R_{\ell}^{\beta}(\mathbf{x}) \geqslant 0$ and $R_{\ell}^{\eta}(\mathbf{x}) \leqslant 0$ according to (27), the sign of each summand in (36) is determined by that of (i) $\Lambda_{\ell}^{\beta}(k)$ or $\Lambda_{\ell}^{\eta}(k)$, and (ii) products $\hat{\beta}^{\prime} \hat{\beta}, \hat{\eta}^{\prime} \hat{\beta}, \hat{\beta}^{\prime} \hat{\eta}$, or $\hat{\eta}^{\prime} \hat{\eta}$. Considering for instance an obstacle characterized by $\beta=1$ and $\eta \neq 1$, the weak scatterer approximation of topological sensitivity (36) reduces, assuming further $\hat{\beta}=1$, to

$$
\mathcal{T}_{\ell}(\mathbf{x}, \beta, \eta)=Q_{\ell} R_{\ell}^{\eta}(\mathbf{x}) \Lambda_{\ell}^{\eta}(k) \hat{\eta}^{\prime} \hat{\eta}+o(|\hat{\eta}|)
$$



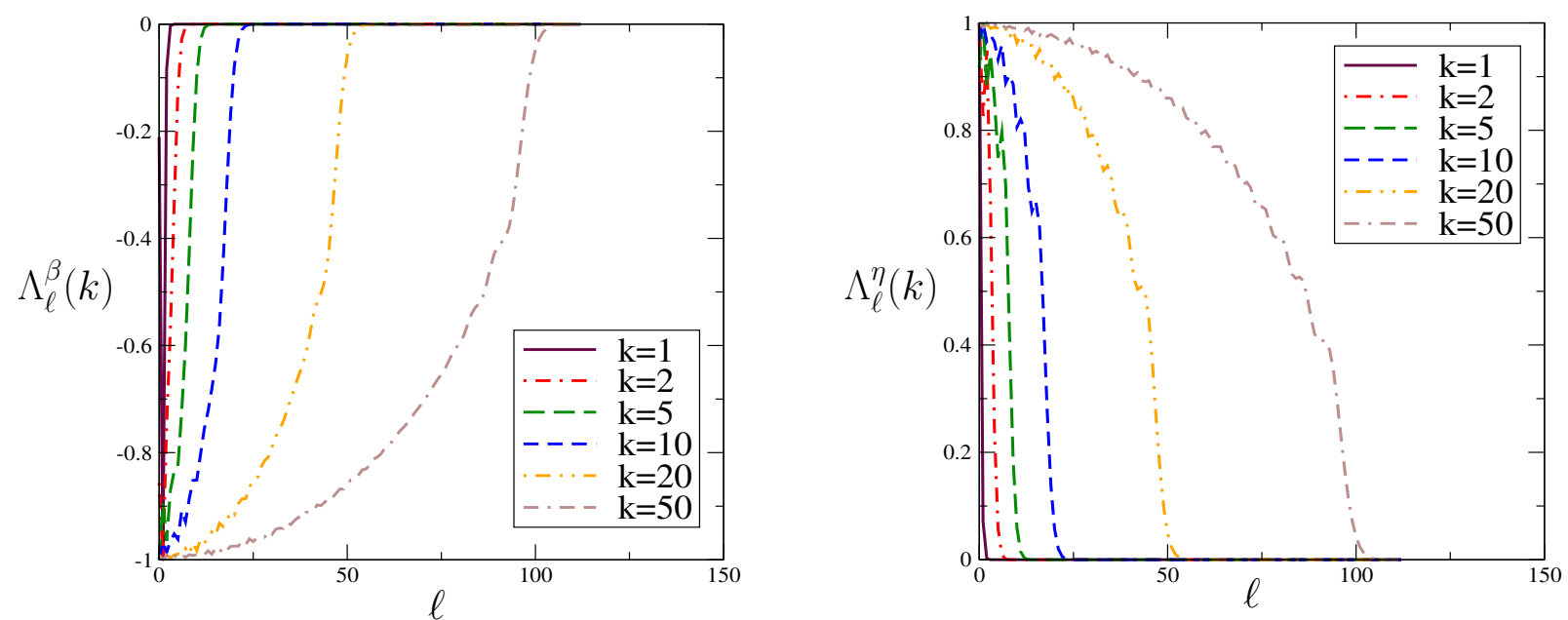

Figure 2: Normalized values of $\Lambda_{\ell}^{\beta}(k)$ and $\Lambda_{\ell}^{\eta}(k)$ for sample values of the wavenumber $k$.

and the usual heuristic underlying the MTS holds in particular if $\Lambda_{\ell}^{\eta}(k) \geqslant 0$ for all $\ell$. Likewise, in situations where $\eta=1$ and $\beta \neq 1$, one finds (assuming $\hat{\eta}=1$ ) that

$$
\mathcal{T}_{\ell}(\mathbf{x}, \beta, \eta)=Q_{\ell} R_{\ell}^{\beta}(\mathbf{x}) \Lambda_{\ell}^{\beta}(k) \frac{3 \hat{\beta}^{\prime} \hat{\beta}}{3+\hat{\beta}^{\prime}}+o(|\hat{\beta}|),
$$

in which case $\Lambda_{\ell}^{\beta}(k) \leqslant 0$ for all $\ell$ will ensure, again, that the heuristic is verified. For completeness, the numerical values of coefficients $\Lambda_{\ell}^{\beta}(k)$ and $\Lambda_{\ell}^{\eta}(k)$, shown in Fig. 2 for a set of wavenumbers $k$, are found to uniformly meet the above conditions on their signs (namely, the $\Lambda_{\ell}^{\beta}(k)$ and $\Lambda_{\ell}^{\eta}(k)$ are all found to be respectively negative and positive).

\subsection{Numerical results}

Obstacle reconstruction. In what follows, the topological derivative $\mathcal{T}\left(\mathbf{x}, \beta^{\prime}, \eta^{\prime}\right)$ is first computed assuming the material properties of the obstacle to be known beforehand, in which case $\beta^{\prime}=\beta$ and $\eta^{\prime}=\eta$. On recalling that: i) the set of incident plane-wave directions is given by $\Sigma=\left\{\boldsymbol{\delta} \in \mathbb{R}^{3},|\boldsymbol{\delta}|=1\right\}$, and ii) the observation surface is a sphere concentric with the ball-shaped obstacle, one finds that the inverse problem, and thus the spatial distribution of $\mathcal{T}$, is spherically symmetric. Accordingly, $\mathcal{T}(\mathbf{x}, \beta, \eta)$ can be completely described via its radial distribution.

In Fig. 3, the dipole and monopole components $\left(\mathcal{T}^{\beta}\right.$ and $\left.\mathcal{T}^{\eta}\right)$ of $\mathcal{T}$ are individually plotted versus radial distance to the sampling point, $|\mathbf{x}|$, for three example wavenumbers of the incident field $(k=5,10,20)$ and two extreme obstacle configurations, namely $\sqrt{\beta \eta}=10^{3}$ (Fig. 3a) and $\sqrt{\beta \eta}=10^{-3}$ (Fig. 3b). In Fig. 3a, describing the case of a (nearly) sound-soft obstacle, one observes that $\mathcal{T}^{\beta}$ attains its maximum negative value inside the obstacle $(|\mathbf{x}|<1)$ while $\mathcal{T}^{\eta}$ has its minimum located outside of $B(|\mathbf{x}|>1)$. Moreover, as the frequency increases, these global minima approach the boundary of the obstacle. This is consistent with a qualitative description of the sound-soft (direct) scattering problem in Section 2, in which an inversion approach exploiting the velocity field is expected to image the interior of the obstacle (nonzero velocity potential inside $B$ ), while using the pressure field should emphasize the boundary of $B$ (vanishing pressure on $\partial B$ and in $B$ ). In contrast, the expected behaviors of $\mathcal{T}^{\beta}$ and $\mathcal{T}^{\eta}$ are reversed for the scattering by a sound-hard obstacle, which is again consistent with the trends observed in Fig. 3b.

As can be seen from Fig. 3 the topological sensitivity provides, despite its complex dependence on the radius and material parameters of the hidden obstacle, a reliable tool for 

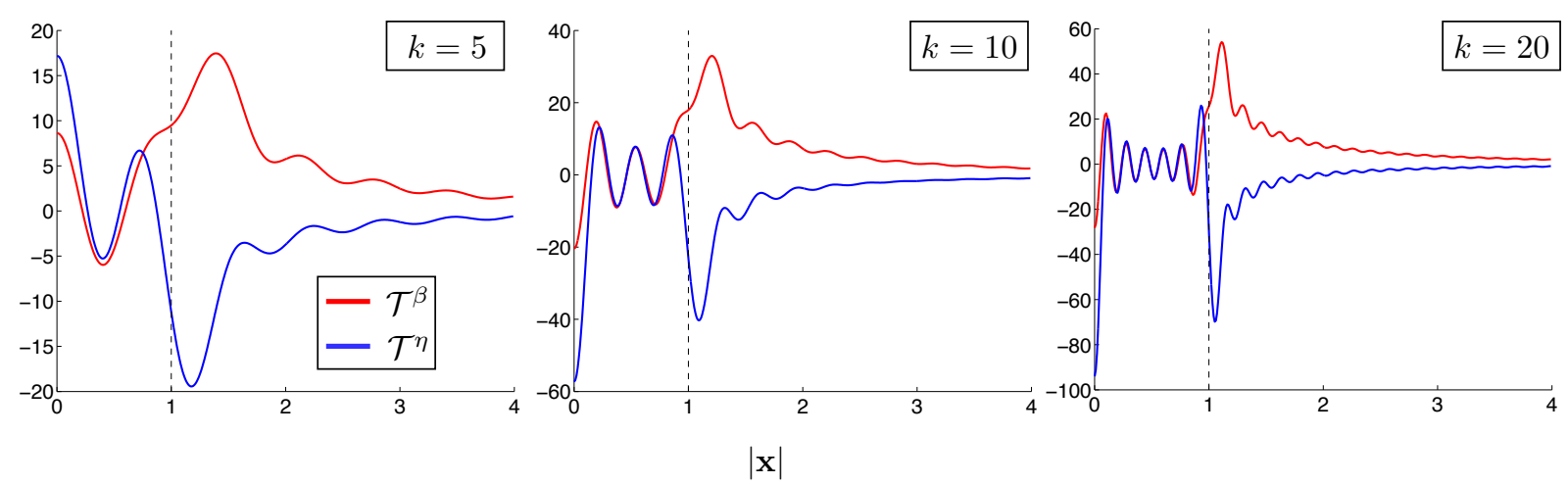

(a) $\beta=10^{3} \quad \eta=10^{3}$
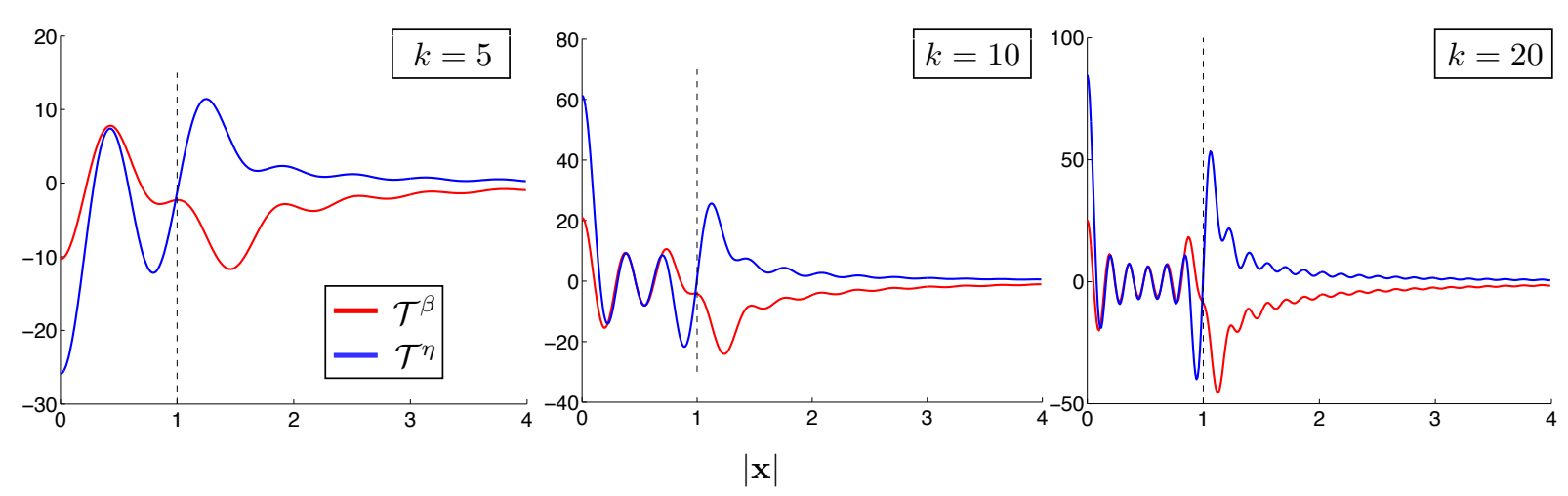

(b) $\beta=10^{-3} \quad \eta=10^{-3}$

Figure 3: Radial distribution of the topological sensitivity components $\mathcal{T}^{\beta}$ and $\mathcal{T}^{\eta}$ for "soundsoft" obstacle (top panels) and "sound-hard" obstacle (bottom panels).
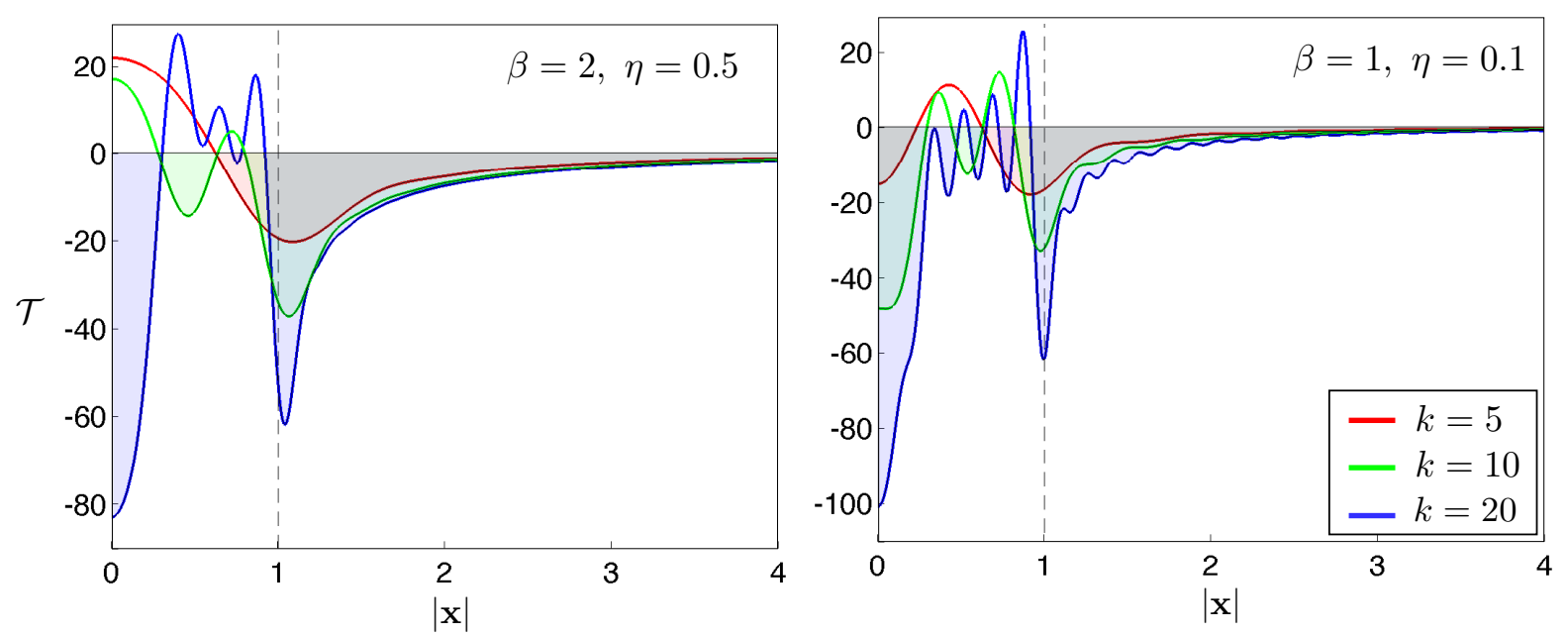

Figure 4: Radial distribution of the topological sensitivity, $\mathcal{T}$, for sample values of the wavenumber $k$ and material parameters $(\beta, \eta)$.

identifying the obstacle boundary as the frequency increases. This claim is further supported via the results for two "moderate" obstacle configurations $(\sqrt{\beta \eta}=1$ and $\sqrt{\beta \eta} \approx 0.32)$ shown in Fig. 4. Moreover, in both Figs. 3 and 4, the magnitude of $|\mathcal{T}|$ exhibits the expected decay as $|\mathbf{x}|$ becomes large, thus aiding the reconstruction by providing the contrast between the neighbourhood of an obstacle and remote regions.

In practice, the obstacle reconstruction via the MTS is effected with the aid of a suitable threshold parameter, $0 \leqslant \alpha \leqslant 1$, whereby the region $B_{\mathrm{MTS}}:=\left\{\mathbf{x} \in \mathbb{R}^{3}, \mathcal{T}(\mathbf{x})<\alpha \min _{\boldsymbol{\xi}} \mathcal{T}\right\}$ is considered to have significant intersection with $B$. From Fig. 4 , it can be seen that $\mathcal{T}$ and thus 
$B_{\text {MTS }}$ at higher frequencies notably highlight the boundary of an obstacle, an observation made in a number of prior studies, see e.g. [24].

Material sensitivity. The MTS is based on the asymptotic expansion corresponding to the nucleation of an infinitesimal scatterer, whose trial properties $\left(\beta^{\prime}, \eta^{\prime}\right)$ may or may not match those $(\beta, \eta)$ of the "true" obstacle. While the exact match $\left(\beta^{\prime}, \eta^{\prime}\right)=(\beta, \eta)$ - which assumes significant prior information - is likely to improve the quality of obstacle reconstruction, the dependence of $\mathcal{T}$ on $\left(\beta^{\prime}, \eta^{\prime}\right)$ can also be seen as a potential tool for material identification [28]. From another point of view, pursued in [35] in the context of transient acoustic waveforms, one can investigate the effect of incompatibility between $\left(\beta^{\prime}, \eta^{\prime}\right)$ and $(\beta, \eta)$ on the quality of obstacle identification. Since the heuristic underlying the MTS rests upon $\mathcal{T}\left(\mathbf{x}, \beta^{\prime}, \eta^{\prime}\right)$ achieving the negative sign at sampling points $\mathbf{x}$ inside (or close to) $B$, it is instructive to examine how the correctness of the sign of $\mathcal{T}\left(\mathbf{x}, \beta^{\prime}, \eta^{\prime}\right)$ is affected by that of coefficients $\left(1-\beta^{\prime}\right)$ and $\left(1-\eta^{\prime}\right)$ relative to $(1-\beta)$ and $(1-\eta)$, respectively. To this end, consider the indicator function $\mathcal{I}\left(\beta^{\prime}, \eta^{\prime}\right)$ defined by

$$
\mathcal{I}\left(\beta^{\prime}, \eta^{\prime}\right)=\frac{1}{n} \sum_{j=1}^{n} \operatorname{sign}\left(\frac{\mathcal{T}\left(\mathbf{x}_{j}, \beta^{\prime}, \eta^{\prime}\right)}{\mathcal{T}\left(\mathbf{x}_{j}, \beta, \eta\right)}\right),
$$

for given a set of sampling points $\mathbb{S}:=\left\{\mathbf{x}_{1}, \ldots, \mathbf{x}_{n}\right\}$, where $\operatorname{sign}(\alpha)=\alpha /|\alpha|$. Thus $-1 \leqslant \mathcal{I} \leqslant 1$, where the limiting value of 1 (resp. -1 ) corresponds to consistently identical (resp. opposite) signs of $\mathcal{T}\left(\mathbf{x}_{j}, \beta^{\prime}, \eta^{\prime}\right)$ and $\mathcal{T}\left(\mathbf{x}_{j}, \beta, \eta\right)$ for all sampling points. In contrast, the values of $\mathcal{I}\left(\beta^{\prime}, \eta^{\prime}\right)$ close to zero indicate numerous sign differences between $\mathcal{T}\left(\mathbf{x}_{j}, \beta^{\prime}, \eta^{\prime}\right)$ and $\mathcal{T}\left(\mathbf{x}_{j}, \beta, \eta\right)$. In this setting, the coefficients in (14) suggest using the parametrization

$$
\mathcal{I}\left(\beta^{\prime}, \eta^{\prime}\right):=\hat{\mathcal{I}}(x, y), \quad x=\frac{\left(1-\beta^{\prime}\right)}{\left(2+\beta^{\prime}\right)} \frac{(2+\beta)}{(1-\beta)}, \quad y=\frac{\left(1-\eta^{\prime}\right)}{(1-\eta)} .
$$

Fig. 5 shows the contour plots of $\hat{\mathcal{I}}(x, y)$ over the parametric region $[-4,4] \times[-4,4]$ for $k=5$ and four choices of $(\beta, \eta)$. In particular, the top left panel $(\beta=2, \eta=0.5)$ corresponds to the case examined in [35], and recovers the observation made therein that $\mathcal{I}(\beta, \eta) \simeq 1$ for $x>0$ and $y>0$, whereas $\mathcal{I}(\beta, \eta) \simeq-1$ for $x<0$ and $y<0$. This observation likewise applies to the results in the top right panel $(\beta=0.5, \eta=2)$, albeit with slightly more variation in the first and the third quadrant. On the other hand, the bottom left panel $(\beta=4, \eta=2)$ highlights the case where $\mathcal{I}(\beta, \eta) \gtrless 0$ for $x>0$ and $y>0$, suggesting a moderation of the statement proposed in [35]. Finally, the bottom right panel $(\beta=0.5, \eta=0.1)$ illustrates the situation where $\mathcal{I}(\beta, \eta) \simeq 1$ within only a narrow sector in the $x-y$ plane.

The above parametric study suggests that the heuristic underlying the MTS (wherein the obstacle is exposed via pronounced negative values of $\mathcal{T}$ ) remains valid with incorrect trial parameters $\left(\beta^{\prime} \neq \beta, \eta^{\prime} \neq \eta\right)$, provided that the material coefficients $x$ and $y$ introduced in (38) are both positive and approximately equal. In light of the physical meaning of $\beta$ (mass density ratio) and $\eta$ (bulk modulus ratio), the previous statement means that the identification of $B$ is qualitatively correct if the material behavior of an infinitesimal obstacle $\left(B_{\mathbf{x}, \varepsilon}, \beta^{\prime}, \eta^{\prime}\right)$ used to derive the asymptotic expansion (6) qualitatively matches that of the "true" scatterer. For completeness, it is noted that the featured sensitivity of the (frequency-domain) MTS to the choice $\beta^{\prime}$ and $\eta^{\prime}$ can be moderated by both multi-modal obstacle illumination and time-domain formulation of the method of topological sensitivity $[9,35]$.

\section{Linear sampling method: spherical obstacle}

Singular value decomposition of operator $\mathcal{S}$. As mentioned earlier, linear operator $\mathcal{S}$ : $L^{2}(\Sigma) \rightarrow L^{2}\left(S_{\text {obs }}\right)$ defined in (15) is compact [34]. Moreover, $\left(Y_{\ell}^{m}\right)_{\ell \in \mathbb{N},-\ell \leqslant m \leqslant+\ell}$ constitute a basis 

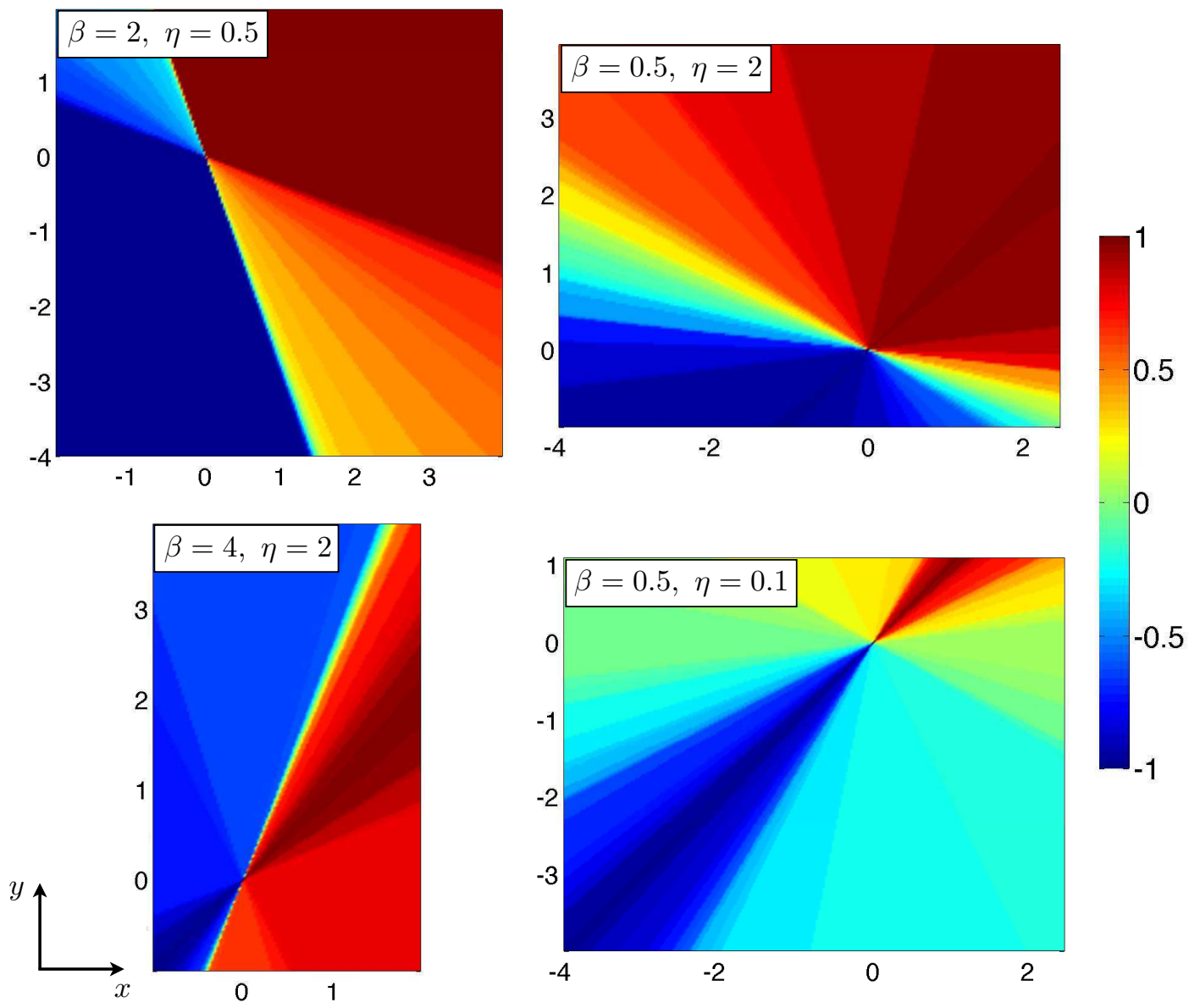

Figure 5: Index $\hat{\mathcal{I}}(x, y)$, given by (38), for sample choices of the "true" material parameters $(\beta, \eta)$.

in $L^{2}(\Sigma)$, while $\left(h_{\ell} Y_{\ell}^{m}\right)_{\ell \in \mathbb{N},-\ell \leqslant m \leqslant+\ell}$ constitute a basis for the outgoing Helmholtz solutions in $\mathbb{R}^{3}$, see [40]. As a result, when restricted to $S_{\text {obs }},\left(h_{\ell} Y_{\ell}^{m}\right)_{\ell \in \mathbb{N},-\ell \leqslant m \leqslant+\ell}$ is a basis of restrictions to $L^{2}\left(S_{\mathrm{obs}}\right)$ of such solutions. Assuming again noise-free data, one has $u_{\mathrm{obs}}=u_{\mathrm{s}}$ on $S_{\text {obs }}$. On inserting expression (23) for $u_{\mathrm{s}}$ into (15), one finds with the aid of the orthonormality property (62) and addition theorem (63) that

$$
\left[\mathcal{S} Y_{\ell}^{m}\right](\boldsymbol{\xi})=4 \pi \mathrm{i}^{\ell} \Lambda_{\ell}(\beta, \eta ; k) h_{\ell}(k R) Y_{\ell}^{m}(\hat{\boldsymbol{\xi}}) \quad\left(\boldsymbol{\xi} \in S_{\mathrm{obs}}, \ell \in \mathbb{N},-\ell \leq m \leq \ell\right) .
$$

Thus, the distinct singular values $\left(\sigma_{\ell}\right)_{\ell \in \mathbb{N}}$ of $\mathcal{S}$ are

$$
\sigma_{\ell}=4 \pi \mathrm{i}^{\ell} \Lambda_{\ell}(\beta, \eta ; k) \quad(\ell \in \mathbb{N}),
$$

which have multiplicity $2 \ell+1$. By way of (24) and large-order asymptotic formulae for the spherical Bessel and Hankel functions given in Appendix B, one can show that $\sigma_{\ell}$ behave asymptotically as

$$
\sigma_{\ell}=\pi \frac{1-\beta}{1+\beta} \frac{k}{\ell}\left(\frac{e k}{2 \ell}\right)^{2 \ell} \mathrm{i}^{\ell+1}\left(1+O\left(\ell^{-1}\right)\right) \quad(\ell \rightarrow \infty)
$$

Clearly, $\sigma_{\ell}$ are characterized by a faster-than-exponential decay with $\ell$ that is independent of $\beta$ and $\eta$; however this decay occurs only for $\ell>\ell_{0}$, where $\ell_{0}$ is a frequency-dependent threshold such that $e k /\left(2 \ell_{0}\right)<1$ as illustrated in Fig. 6 . 


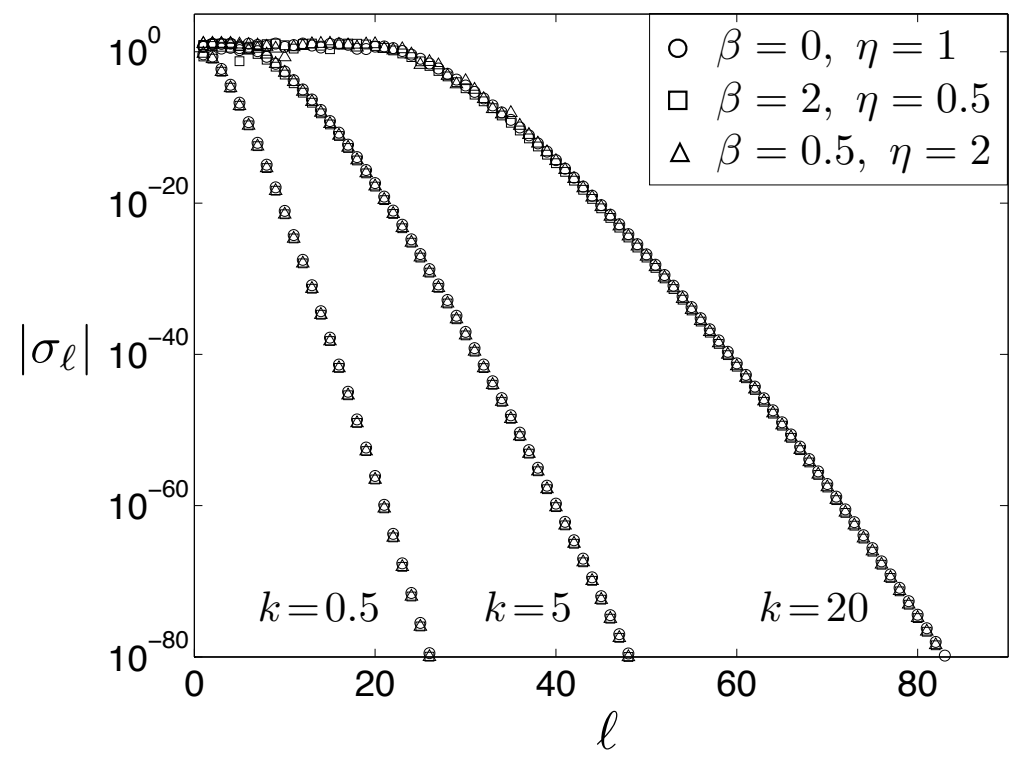

Figure 6: Singular value spectrum of the LSM operator for sample values of the wavenumber $k$ and material parameters $(\beta, \eta)$.

Interior transmission problem. As indicated in Sec. 2.1.2, integral equation (16) underlying the LSM is solvable provided that $k$ is not a transmission eigenvalue of (17). For the purpose of this study, these eigenvalues are next investigated for the specific problem at hand, by seeking the conditions under which the interior transmission problem (17), with $B$ chosen as the unit ball, has non-trivial solution $(v, w)$ such that $v$ is a Herglotz wave function (i.e. representable as (18) for some density $f \in L^{2}(\Sigma)$ ). Following (22), the featured solution pair is sought in the form of spherical harmonics, namely

$$
\begin{aligned}
v(\boldsymbol{\xi}) & =\sum_{\ell=0}^{+\infty} \sum_{m=-\ell}^{+\ell} v_{\ell}^{m} j_{\ell}(k|\boldsymbol{\xi}|) Y_{\ell}^{m}(\hat{\boldsymbol{\xi}}) \quad(\boldsymbol{\xi} \in B), \\
w(\boldsymbol{\xi}) & =\sum_{\ell=0}^{+\infty} \sum_{m=-\ell}^{+\ell} w_{\ell}^{m} j_{\ell}(\gamma k|\boldsymbol{\xi}|) Y_{\ell}^{m}(\hat{\boldsymbol{\xi}}) \quad(\boldsymbol{\xi} \in B) .
\end{aligned}
$$

By virtue of the Funk-Hecke identity (67), any $v$ of the above form is a Herglotz wave function. On enforcing the boundary conditions (17c) and (17d), one finds from (42) that a non-trivial solution pair $(v, w)$ exists for all wavenumbers $k$ such that

$$
\alpha_{\ell_{0}} j_{\ell_{0}}(k)=j_{\ell_{0}}^{\prime}(k), \quad \ell_{0} \in \mathbb{N},
$$

where $\alpha_{\ell_{0}}(\beta, \eta)$ is defined in (24). Condition (43) in particular implies that $\Lambda_{\ell_{0}}(\beta, \eta ; k)=0$, i.e that the scattered field induced by an incident field of the form of a Herglotz wave function (18) with density $f(\boldsymbol{\delta})=Y_{\ell_{0}}^{m}(\boldsymbol{\delta})$ vanishes, making the unit penetrable ball $B$ undetectable for that particular excitation.

To show that such transmission eigenvalues exist (i.e. that the characteristic equation (43) is verified for some values of $k$ and $\ell_{0}$ ), it is sufficient to consider the case $\ell_{0}=0$, which implies spherically-symmetric eigenfunctions of the form $v(\boldsymbol{\xi})=v_{0} j_{0}(k|\boldsymbol{\xi}|)$ and $w(\boldsymbol{\xi})=w_{0} j_{0}(\gamma k|\boldsymbol{\xi}|)$; a result that can also be obtained from the study on stratified spherical obstacles [22] as a degenerate case. As an illustration, characteristic function $\Delta(k ; \beta, \eta):=\alpha_{0}(\beta, \eta) j_{0}(k)-j_{0}^{\prime}(k)$ is plotted versus $k$ for two choices of $(\beta, \eta)$ in Fig. 7. Clearly, $\Delta=0$ for several frequencies within the range shown, providing (numerical) evidence of the existence of such eigenvalues. For completeness, it is noted that it is possible to show that the interior transmission eigenvalues form a countable set, see e.g. [22]. 


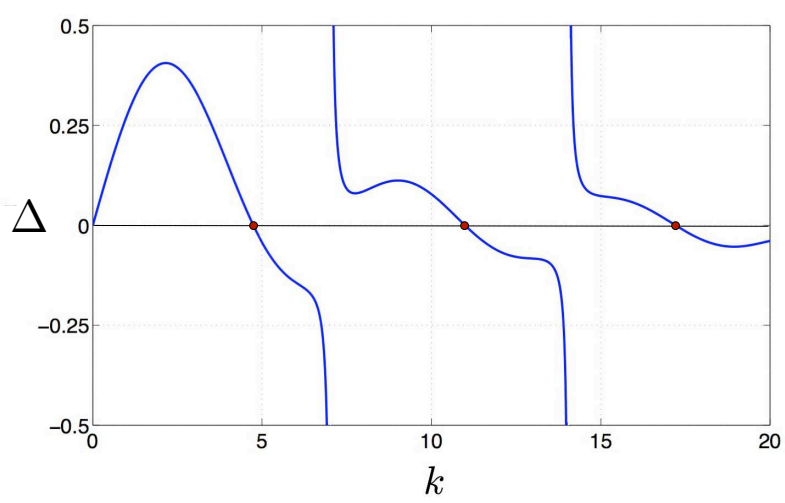

(a) $\beta=0.5 \quad \eta=0.1$

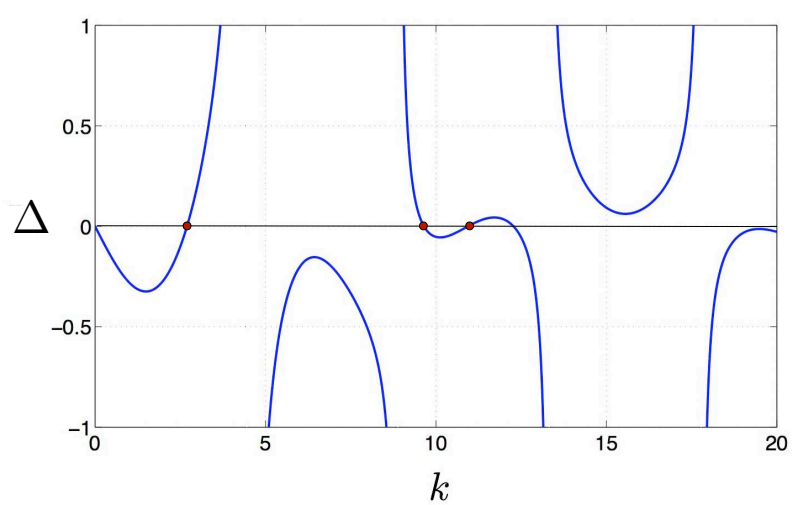

(b) $\beta=4 \quad \eta=2$

Figure 7: LSM characteristic function $\Delta(k ; \beta, \eta)$ versus the excitation wavenumber.

Analytical expression. By virtue of the spherical-harmonics expansion (23) of $u_{\mathrm{s}}$, the indicator function $g(\mathbf{x}, \cdot)$ featured in the linear sampling equation (16) can now be computed analytically provided that $k$ is not a transmission eigenvalue. For a given sampling point $\mathbf{x} \in B_{R}, g(\mathbf{x}, \cdot) \in L^{2}(\Sigma)$ can be expanded in terms of spherical harmonics as

$$
g(\mathbf{x}, \boldsymbol{\delta})=\sum_{\ell=0}^{+\infty} \sum_{m=-\ell}^{+\ell} g_{\ell}^{m}(\mathbf{x}) Y_{\ell}^{m}(\boldsymbol{\delta}) \quad(\boldsymbol{\delta} \in \Sigma)
$$

On substituting (39) and expansion (69) of $G(\boldsymbol{\xi}, \mathbf{x})$ in spherical harmonics into the LSM integral equation (16), the coefficients $g_{\ell}^{m}(\mathbf{x})$ of expansion (44) are readily found as

$$
g_{\ell}^{m}(\mathbf{x})=\frac{k}{(4 \pi)} \frac{j_{\ell}(k|\mathbf{x}|)}{\mathrm{i}^{\ell-1} \Lambda_{\ell}(\beta, \eta ; k)} \bar{Y}_{\ell}^{m}(\mathbf{x}),
$$

noting that $\Lambda_{\ell}(\beta, \eta ; k) \neq 0, \forall \ell \in \mathbb{N}$ since $k$ is assumed to differ from a transmission eigenvalue.

On the other hand, with the help of the asymptotic formulae for the spherical Bessel and Hankel functions (see Appendix B) and addition theorem (63), the large-order behavior of $g_{\ell}^{m}(\mathbf{x})$ is such that

$$
\sum_{m=-\ell}^{\ell}\left|g_{\ell}^{m}(\mathbf{x})\right|^{2}=\frac{k^{2}}{(4 \pi)^{3}}(2 \ell+1)\left(\frac{j_{\ell}(k|\mathbf{x}|)}{\left|\Lambda_{\ell}(\beta, \eta ; k)\right|}\right)^{2} \underset{\ell \rightarrow \infty}{=} \frac{\ell}{16 \pi^{3}}\left(\frac{1+\beta}{1-\beta}\right)^{2}\left(\frac{2 \ell|\mathbf{x}|}{e k}\right)^{2 \ell}\left(1+O\left(\ell^{-1}\right)\right),
$$

from which it follows that the $L^{2}(\Sigma)$-norm of the indicator function $g(\mathbf{x}, \boldsymbol{\delta})$ defined by $(44)$ and (45) blows up, i.e. $\|g(\mathbf{x}, \cdot)\|_{L^{2}(\Sigma)}=+\infty$ for $|\mathbf{x}|>0$. This result is not surprising since the singular values (40) of operator $\mathcal{S}$ accumulate at zero with faster-than-exponential decay with respect to $\ell$. The blow-up of the $L^{2}(\Sigma)$-norm of $g(\mathbf{x}, \cdot)$ can therefore be attributed to the smallest singular values. Following the approach in [17] for electromagnetism, a regularized solution $g_{L}(\mathbf{x}, \cdot)$ to the integral equation (16) is instead defined by truncating the series representation (44) as

$$
g_{L}(\mathbf{x}, \boldsymbol{\delta})=\frac{k}{(4 \pi)^{2}} \sum_{\ell=0}^{L} \frac{(2 \ell+1)}{\mathrm{i}^{\ell-1} \Lambda_{\ell}(\beta, \eta ; k)} j_{\ell}(k|\mathbf{x}|) P_{\ell}(\hat{\mathbf{x}} \cdot \boldsymbol{\delta}) \quad(\boldsymbol{\delta} \in \Sigma),
$$

which makes use of (63). In what follows, $L$ is selected such the singular value spectrum (40) of the truncated operator $\mathcal{S}$ has a prescribed infimum. With reference to (21) the LSM finally consists, for the present configuration, in evaluating the indicator function

$$
I_{L}(\mathbf{x}):=\frac{1}{\left\|g_{L}(\mathbf{x}, \cdot)\right\|_{L^{2}(\Sigma)}} \quad\left(\mathbf{x} \in B_{R}\right)
$$




\subsection{Numerical results}

Fig. 8 plots the indicator function $I_{L}(\mathbf{x})$ versus radial distance to the sampling coordinate, $|\mathbf{x}|$, with the truncation index $L$ chosen so that $\left|\sigma_{\ell}\right|>10^{-10}, \quad \ell \leqslant L$. In contrast to the MTS approach, the LSM voids the need for prior information on the material properties $(\beta$ and $\eta)$ of a hidden obstacle. As highlighted by Fig. 8, these material properties somewhat influence the indicator function, which otherwise behaves as expected from the theory (see Sec. 2.1.2 and Sec. 4). Moreover the transition region appears to be frequency-dependent, the identified location of $\partial B$ being insufficiently accurate for a low frequency $(k=5)$ but quite reasonable using a higher frequency $(k=20)$.
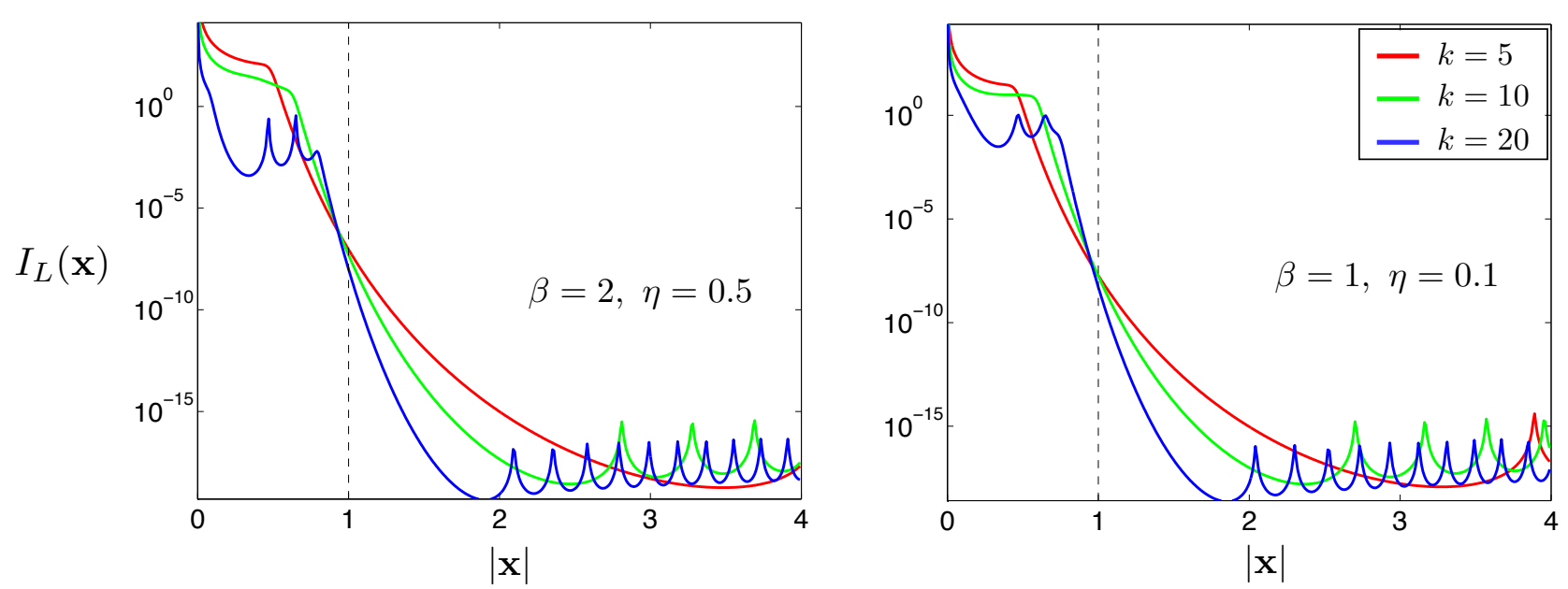

Figure 8: Radial variation of the LSM indicator function for sample values of the wavenumber $k$ and material parameters $(\beta, \eta)$.

\subsection{Factorization method}

The factorization method (FM), see [32], is a variant of the LSM where one considers the solvability of the equation

$$
\left[\left(\mathcal{S}^{\star} \mathcal{S}\right)^{1 / 4} g(\mathbf{x}, \cdot)\right](\hat{\boldsymbol{\xi}})=G_{\infty}(\hat{\boldsymbol{\xi}}, \mathbf{x}) \quad\left(\mathbf{x} \in \mathbb{R}^{3}, \hat{\boldsymbol{\xi}} \in \Sigma\right),
$$

in terms of $g \in L^{2}(\Sigma)$, where

$$
G_{\infty}(\hat{\boldsymbol{\xi}}, \mathbf{x}):=\exp (-\mathrm{i} k \mathbf{x} \cdot \hat{\boldsymbol{\xi}})
$$

denotes the far-field pattern of the fundamental solution (10). In this setting, one can show [32] that

$$
\mathbf{x} \in B \quad \text { if and only if } \quad\|g(\mathbf{x}, \cdot)\|_{L^{2}(\Sigma)}^{2}<+\infty,
$$

i.e. that $I_{F M}(\mathbf{x}):=\left(\|g(\mathbf{x}, \cdot)\|_{L^{2}(\Sigma)}\right)^{-1}$ can be considered as the indicator function for the FM. On applying the definition

$$
\left(\mathcal{S}^{\star} g, h\right)_{L^{2}\left(\Sigma_{R}\right)}=(g, \mathcal{S} h)_{L^{2}(\Sigma)} \quad\left(g \in L^{2}(\Sigma), h \in L^{2}\left(\Sigma_{R}\right)\right)
$$

of the adjoint operator $\mathcal{S}^{\star}$ where $\mathcal{S}$ is given by (39), one finds that

$$
\begin{aligned}
{\left[\left(\mathcal{S}^{\star} \mathcal{S}\right) Y_{\ell}^{m}\right](\hat{\boldsymbol{\xi}}) } & =(4 \pi R)^{2}\left|\Lambda_{\ell}(\beta, \eta ; k)\right|^{2}\left|h_{\ell}(k R)\right|^{2}, \\
{\left[\left(\mathcal{S}^{\star} \mathcal{S}\right)^{1 / 4} Y_{\ell}^{m}\right](\hat{\boldsymbol{\xi}}) } & =(4 \pi R)^{1 / 2}\left|\Lambda_{\ell}(\beta, \eta ; k)\right|^{1 / 2}\left|h_{\ell}(k R)\right|^{1 / 2} .
\end{aligned}
$$


Upon applying the Jacobi-Anger expansion (66) to the definition of $G_{\infty}(\hat{\boldsymbol{\xi}}, \mathbf{x})$, the solution of (48), namely $g(\mathbf{x}, \hat{\boldsymbol{\xi}})=\sum_{\ell=0}^{+\infty} \sum_{m=-\ell}^{\ell} g_{\ell}^{m}(\mathbf{x}) Y_{\ell}^{m}(\hat{\boldsymbol{\xi}})$ is found to read

$$
g_{\ell}^{m}(\mathbf{x})=\left(\frac{4 \pi}{R}\right)^{1 / 2} \frac{j_{\ell}(k|\mathbf{x}|) \bar{Y}_{\ell}^{m}(\hat{\boldsymbol{\xi}})}{\left|\Lambda_{\ell}(\beta, \eta ; k)\right|^{1 / 2}\left|h_{\ell}(k R)\right|^{1 / 2}} .
$$

By virtue of this result and (63), the solvability condition (50) becomes

$$
\frac{2 \ell+1}{R} \sum_{\ell=0}^{+\infty} \frac{j_{\ell}^{2}(k|\mathbf{x}|)}{\left|\Lambda_{\ell}(\beta, \eta ; k)\right|\left|h_{\ell}(k R)\right|}<+\infty .
$$

Here it is useful to note from (28b) and large-order asymptotic formulae in Appendix B that

$$
\frac{j_{\ell}^{2}(k|\mathbf{x}|)}{\left|\Lambda_{\ell}(\beta, \eta ; k)\right|}=\frac{1}{2 k \ell} \frac{1+\beta}{1-\beta}|\mathbf{x}|^{2 \ell}\left(1+O\left(\ell^{-1}\right)\right) \quad(\ell \rightarrow \infty) .
$$

In view of the large-order behavior $(70)$ of $h_{\ell}(k R)$, condition (52) seems to be verified for any sampling point $\mathbf{x}$. However, the factorization method in fact requires that measurements be taken at infinity (i.e. assumes that the data consists of the far-field pattern of the scattered field), which corresponds to the limiting case $R \rightarrow \infty$ in the expression (51) for $\mathcal{S}^{\star} \mathcal{S}$. In that case, one has $k R\left|h_{\ell}(k R)\right| \rightarrow 1$ [1], and condition (52) becomes

$$
\sum_{\ell=0}^{+\infty} \frac{1+\beta}{1-\beta}|\mathbf{x}|^{2 \ell}<+\infty
$$

Since the above series is convergent for $|\mathbf{x}|<1$ and divergent otherwise one finds, for the case of a spherical inclusion, that the solvability condition (50) exactly determines the geometry of the scatterer, as predicted by the theory [32]. Unlike the LSM, no regularization is necessary for defining the indicator function $I_{F}(\mathbf{x})$. The conditions for the application of the FM are however more restrictive, since the latter requires the far-field data (and corresponding far-field version of the observation operator $\mathcal{S}$ ) whereas the LSM is applicable to both far- and near-field data.

\section{$5 \quad$ Effect of noise in the data}

The foregoing analytical developments for both the MTS and the LSM assume perfect, noisefree data $u_{\mathrm{obs}}=u_{\mathrm{s}}$ on $S_{\text {obs. }}$. However, practical identification problems invariably involve noisy data. In this section, the sensitivity of both the MTS and the LSM to data noise is investigated. To this end, let the data $u_{\text {obs }}$ on $S_{\text {obs }}$ be contaminated by additive noise distribution $\nu$, namely

$$
\tilde{u}_{\mathrm{s}}(\boldsymbol{\xi}, \boldsymbol{\delta})=u_{\mathrm{s}}(\boldsymbol{\xi}, \boldsymbol{\delta})+\nu(\boldsymbol{\xi}) \quad\left(\boldsymbol{\xi} \in S_{\mathrm{obs}}, \boldsymbol{\delta} \in \Sigma\right) .
$$

\subsection{Method of topological sensitivity}

Let $\varphi$ and $\varphi_{\nu}$ denote the densities of cost functional (4) based on noise-free and noise-contaminated data, respectively. On substituting the adjoint field expression (9) into the MTS formula (8) for both densities, the perturbation of $\mathcal{T}$ due to measurement noise can be written as

$$
\begin{aligned}
& \mathcal{T}_{\nu}\left(\mathbf{x}, \beta^{\prime}, \eta^{\prime}\right)-\mathcal{T}\left(\mathbf{x}, \beta^{\prime}, \eta^{\prime}\right)=\int_{\Sigma} \int_{S_{\mathrm{obs}}} \operatorname{Re}\left\{\left[\partial_{u} \varphi_{\nu}-\partial_{u} \varphi\right](0, \boldsymbol{\zeta}, \boldsymbol{\delta})\right. \\
& {\left.\left[\left(1-\beta^{\prime}\right) \nabla_{1} G(\mathbf{x}, \boldsymbol{\zeta}) \cdot \mathcal{A}\left(\mathcal{B}, \beta^{\prime}, \eta^{\prime}\right) \cdot \nabla u_{\mathrm{i}}(\mathbf{x}, \boldsymbol{\delta})-\left(1-\eta^{\prime}\right) k^{2} G(\mathbf{x}, \boldsymbol{\zeta}) u_{\mathrm{i}}(\mathbf{x}, \boldsymbol{\delta})\right]\right\} \mathrm{d} \boldsymbol{\zeta} \mathrm{d} \boldsymbol{\delta} }
\end{aligned}
$$


where $\nabla_{1}$ denotes the gradient with respect to the first argument. An upper bound for this perturbation can be written as

$$
\left|\mathcal{T}_{\nu}\left(\mathbf{x}, \beta^{\prime}, \eta^{\prime}\right)-\mathcal{T}\left(\mathbf{x}, \beta^{\prime}, \eta^{\prime}\right)\right| \leqslant C\left(\mathbf{x}, \beta^{\prime}, \eta^{\prime}, k\right)\left\|\partial_{u} \varphi_{\nu}-\partial_{u} \varphi\right\|_{L^{2}\left(S_{\mathrm{obs}} \times \Sigma\right)}
$$

where

$$
\left\|\partial_{u} \varphi_{\nu}-\partial_{u} \varphi\right\|_{L^{2}\left(S_{\mathrm{obs}} \times \Sigma\right)}=\left[\int_{\Sigma} \int_{S_{\mathrm{obs}}}\left|\partial_{u} \varphi_{\nu}-\partial_{u} \varphi\right|^{2}(0, \boldsymbol{\zeta}, \boldsymbol{\delta}) \mathrm{d} \boldsymbol{\zeta} \mathrm{d} \boldsymbol{\delta}\right]^{1 / 2}
$$

and

$$
C\left(\mathbf{x}, \beta^{\prime}, \eta^{\prime}, k\right)=c\left(\beta^{\prime}, \eta^{\prime}, k\right)\left(\left\|\nabla_{1} G(\mathbf{x}, \cdot)\right\|_{L^{2}\left(S_{\mathrm{obs}}\right)}\left\|\nabla u_{\mathrm{i}}(\mathbf{x}, \cdot)\right\|_{L^{2}(\Sigma)}+\|G(\mathbf{x}, \cdot)\|_{L^{2}\left(S_{\mathrm{obs}}\right)}\left\|u_{\mathrm{i}}(\mathbf{x}, \cdot)\right\|_{L^{2}(\Sigma)}\right),
$$

where $c$ is a constant dependent on the trial material parameters $\left(\beta^{\prime}, \eta^{\prime}\right)$ and wavenumber $k$. In the case of the least-squares misfit function, one further has

$$
\left\|\partial_{u} \varphi_{\nu}-\partial_{u} \varphi\right\|_{L^{2}\left(S_{\mathrm{obs}} \times \Sigma\right)}=\left\|u_{\mathrm{obs}}-u_{\mathrm{s}}\right\|_{L^{2}\left(S_{\mathrm{obs}} \times \Sigma\right)}=2 \sqrt{\pi}\|\nu\|_{L^{2}\left(S_{\mathrm{obs}}\right)} .
$$

For $\mathbf{x} \notin S_{\text {obs }}$, the coefficient $C$ in (55) is clearly bounded, and so is the perturbation of topological derivative. Expressions (55) and (57) accordingly show that, for the least-squares cost function, the perturbation of $\mathcal{T}$ depends linearly on the measurement noise. In view of the usual ill-posedness of inverse problems, such sensitivity to the noise in the data can be qualified as "benign".

\section{$5.2 \quad$ Linear sampling method}

Consider the LSM equation (16) with the near-field operator $\mathcal{S}$ replaced by its counterpart $\mathcal{S}_{\nu}$, constructed from noise-polluted data (53). Next, let $g_{\nu}(\mathbf{x}, \cdot) \in L^{2}(\Sigma)$ denote the solution of such perturbed equation so that

$$
\left[\mathcal{S}_{\nu} g_{\nu}(\mathbf{x}, \cdot)\right](\boldsymbol{\xi})=\int_{\Sigma} u_{\mathrm{obs}}(\boldsymbol{\xi}, \boldsymbol{\delta}) g_{\nu}(\mathbf{x}, \boldsymbol{\delta}) \mathrm{d} \boldsymbol{\delta}=G(\boldsymbol{\xi}, \mathbf{x}) \quad\left(\boldsymbol{\xi} \in S_{\text {obs }}\right),
$$

where, according to (15) and (53),

$$
\left[\mathcal{S}_{\nu} f\right](\boldsymbol{\xi})=[\mathcal{S} f](\boldsymbol{\xi})+\nu(\boldsymbol{\xi}) \int_{\Sigma} f(\boldsymbol{\delta}) \mathrm{d} \boldsymbol{\delta}
$$

Equations (16) and (58) imply that

$$
\left[\mathcal{S}_{\nu} g_{\nu}(\mathbf{x}, \cdot)\right](\boldsymbol{\xi})=[\mathcal{S} g(\mathbf{x}, \cdot)](\boldsymbol{\xi}) \quad\left(\boldsymbol{\xi} \in S_{\text {obs }}\right) .
$$

On deploying the usual operator norm $[37]$ on $\mathcal{L}\left[L^{2}(\Sigma), L^{2}\left(S_{\text {obs }}\right)\right]$, denoted by $\|\cdot\|_{\text {op }}$, it follows via the inequality $\|\cdot\|_{L^{1}\left(S_{\mathrm{obs}}\right)} \leqslant 2 \sqrt{\pi}\|\cdot\|_{L^{2}\left(S_{\mathrm{obs}}\right)}$ that

$$
\left\|g_{\nu}(\mathbf{x}, \cdot)-g(\mathbf{x}, \cdot)\right\|_{L^{2}(\Sigma)} \leqslant 2 \sqrt{\pi}\left\|\mathcal{S}^{-1}\right\|_{\text {op }}\|\nu\|_{L^{2}\left(S_{\mathrm{obs}}\right)}\left\|g_{\nu}(\mathbf{x}, \cdot)\right\|_{L^{2}(\Sigma)} .
$$

As a result, the relative error in the LSM indicator function (21) due to noise in the data is bounded as

$$
\left|\frac{I_{\mathrm{LSM}, \nu}(\mathbf{x})-I_{\mathrm{LSM}}(\mathbf{x})}{I_{\mathrm{LSM}}(\mathbf{x})}\right|=\frac{\left|\|g(\mathbf{x}, \cdot)\|_{L^{2}(\Sigma)}-\left\|g_{\nu}(\mathbf{x}, \cdot)\right\|_{L^{2}(\Sigma)}\right|}{\left\|g_{\nu}(\mathbf{x}, \cdot)\right\|_{L^{2}(\Sigma)}} \leqslant 2 \sqrt{\pi} \operatorname{Cond}(\mathcal{S}) \frac{\|\nu\|_{L^{2}\left(S_{\mathrm{obs}}\right)}}{\|\mathcal{S}\|_{\mathrm{op}}}
$$

where $\operatorname{Cond}(\mathcal{S})=\|\mathcal{S}\|_{\text {op }}\left\|\mathcal{S}^{-1}\right\|_{\text {op }}$ is the condition number of $\mathcal{S}$. Since the latter is a compact operator, its singular values accumulate at zero whereby $\operatorname{Cond}(\mathcal{S}) \gg 1$. In other words, $\mathcal{S}$ is ill-conditioned, and from (60) it is seen that the indicator function may significantly amplify the noise present in the data. Consequently, any application of the LSM necessitates suitable stabilization of the problem, e.g. in terms of Tikhonov regularization that is successfully employed in many situations [13]. For the unit ball problem examined in this study, such regularization is effected via the use of the truncation index $L$, see (46). 


\subsection{Numerical results}

In what follows, numerical results are presented to illustrate the error estimates (55) and (60). The sample configuration assumes unit spherical obstacle with $\beta=2$ and $\eta=0.5$, illuminated via plane waves at wavenumber $k=10$. The noise in the data is simulated following additive decomposition (53). With reference to the expansion (23) of the scattered field, the noise function $\nu$ is calibrated so that the singular values $\left(\sigma_{\nu, \ell}\right)_{\ell \in \mathbb{N}}$ of the "noisy" near-field operator $\mathcal{S}_{\nu}$ depart from those of its noise-free counterpart $\mathcal{S}$, see (40), according to

$$
\sigma_{\nu, \ell}=\sigma_{\ell}+\epsilon_{\ell} \quad(\ell \in \mathbb{N}),
$$

where the complex-valued random perturbation $\epsilon_{\ell}$ is specified in terms of uniformly distributed random numbers $r_{1}, r_{2} \in \mathbb{R}$ as $\epsilon_{\ell}=\left(r_{1}+\mathrm{i} r_{2}\right)$. This choice provides a consistent framework to describe the effect of noise-polluted data for both MTS and LSM indicator functions. In this setting, the LSM indicator function (47) is computed using the truncation index $L$ chosen so that $\left|\sigma_{\nu, \ell}\right|<\left|\epsilon_{\ell}\right|$ for $\ell>L$. The random distributions $r_{1}$ and $r_{2}$ are adjusted to achieve the relative $L^{2}\left(S_{\text {obs }}\right)$-norm of the error in the data as

$$
\frac{\left\|\tilde{u}_{\mathrm{s}}-u_{\mathrm{s}}\right\|_{L^{2}\left(S_{\mathrm{obs}}\right)}}{\left\|u_{\mathrm{s}}\right\|_{L^{2}\left(S_{\mathrm{obs}}\right)}}=\epsilon .
$$

In Fig. 9, indicator functions $\mathcal{T}$ and $\log \left(I_{L}\right)$ are plotted for sampling points $\mathbf{x}$ spanning the plane $[-4,4] \times[-4,4]$ containing the origin and three sample values $\left(\epsilon=10^{-2}, 5 \cdot 10^{-2}, 10^{-1}\right)$ of the noise level. The figure shows the images obtained by applying the two methods to the noise-polluted data. The reconstruction of the shape of the obstacle, i.e. either its boundary or its interior, remains relatively stable as $\epsilon$ increases. However, the results indicate that the MTS indicator $\mathcal{T}$ is only mildly affected by the noise in the data, whereas its LSM companion $I_{L}$ is more severely perturbed as $\epsilon$ increases - a result that is consistent with the respective error estimates (55) and (60).

$$
\epsilon=10^{-2}
$$

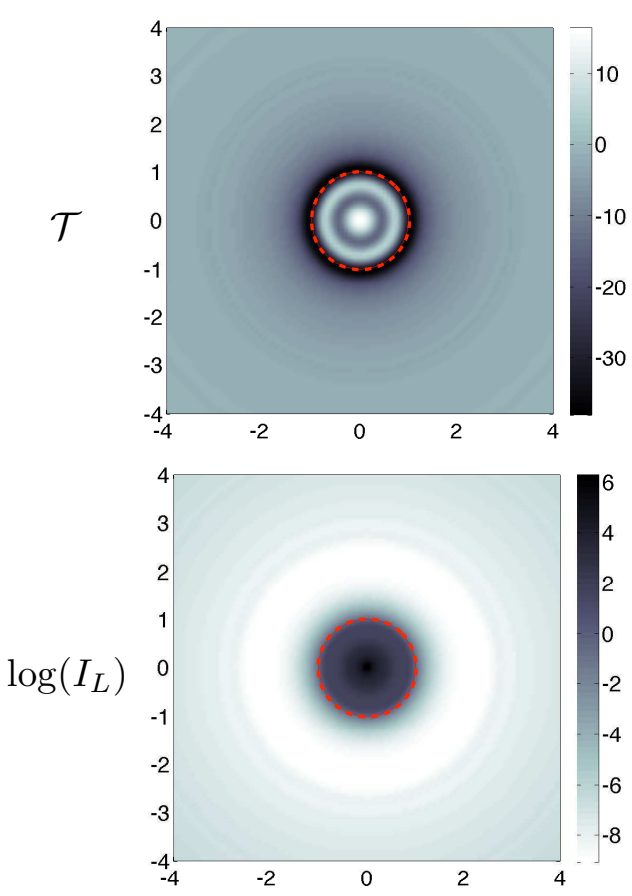

$\epsilon=5 \cdot 10^{-2}$
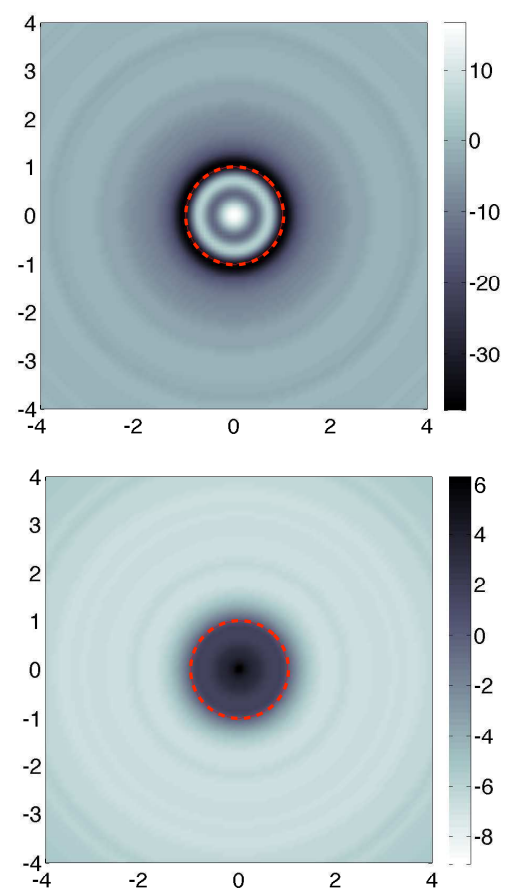

$\epsilon=10^{-1}$
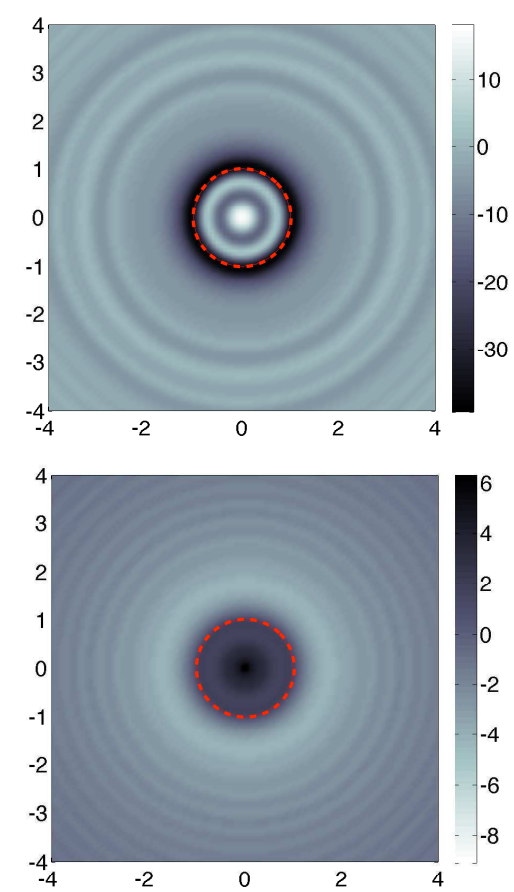

Figure 9: Reconsructions of a unit penetrable ball $(\beta=2, \eta=0.5)$ via the application of the MTS and LSM indicator functions to noise-polluted data $(k=10)$ for three sample values of the relative noise level $\epsilon$. 


\section{Conclusions}

In this study, inverse scattering of scalar waves by a penetrable obstacle is investigated by way of two particular non-iterative reconstruction techniques, namely the method of topological sensitivity (MTS) and the linear sampling method (LSM). To provide a common analytical platform for the assessment and comparison of the two methods, the forward problem of scattering by a penetrable ball, concentric with the observation surface, is solved analytically. In this setting the MTS, affiliated with the heuristic interpretation of an infinitesimal perturbation of a cost functional, is treated explicitly via an adjoint field formulation which results in a series representation of the germane indicator function in terms of spherical Bessel and Hankel functions. Thus obtained solution is used to investigate the critical features of the MTS both analytically, in terms of limiting behavior, and numerically, by exposing the dependence of the solution on the excitation frequency and material parameters involved in the problem. On the other hand the analytical formulation of the LSM, whose performance is supported by key theoretical results, highlights the central role of the interior transmission problem characterizing the (countable set of) frequency values at which the method proves to be ineffective. Excluding the latter set from further consideration, the focus is made on the influence of the frequency of excitation on the resolution of the indicator function. For completeness, an effort is made to characterize and compare the effect of noise in the data, both analytically and numerically, on the performance of the two methods.

\section{A Properties of spherical harmonics}

Spherical harmonics $\left(Y_{\ell}^{m}\right)_{\ell \in \mathbb{N},-\ell \leqslant m \leqslant+\ell}$ are functions defined on the unit sphere, $\Sigma$, that constitute an orthonormal basis of $L^{2}(\Sigma)$. They satisfy the differential equation

$$
\Delta_{\Sigma} Y_{\ell}^{m}(\boldsymbol{\zeta})+\ell(\ell+1) Y_{\ell}^{m}(\boldsymbol{\zeta})=0 \quad(\forall \boldsymbol{\zeta} \in \Sigma, \forall \ell \in \mathbb{N}, \forall m \in\{-\ell, \ldots, \ell\})
$$

where $\Delta_{\Sigma}$ is the angular part of the Laplace operator in spherical coordinates; the orthonormality relations

$$
\int_{\Sigma} Y_{\ell}^{m}(\boldsymbol{\zeta}) \bar{Y}_{\ell^{\prime}}^{m^{\prime}}(\boldsymbol{\zeta}) \mathrm{d} \boldsymbol{\zeta}=\delta_{\ell \ell^{\prime}} \delta_{m m^{\prime}} \quad\left(\ell, \ell^{\prime}\right) \in \mathbb{N}^{2},-\ell \leq m \leq \ell,-\ell^{\prime} \leq m^{\prime} \leq \ell^{\prime}
$$

and the addition theorem

$$
P_{\ell}(\boldsymbol{\xi} \cdot \boldsymbol{\zeta})=\frac{4 \pi}{2 \ell+1} \sum_{m=-\ell}^{+\ell} Y_{\ell}^{m}(\boldsymbol{\xi}) \bar{Y}_{\ell}^{m}(\boldsymbol{\zeta}), \quad(\boldsymbol{\xi}, \boldsymbol{\zeta}) \in \Sigma^{2}
$$

Moreover, since $P_{\ell}(1)=1 \quad \forall \ell \in \mathbb{N}$, the addition theorem implies that

$$
\sum_{m=-\ell}^{+\ell} Y_{\ell}^{m}(\boldsymbol{\zeta}) \bar{Y}_{\ell}^{m}(\boldsymbol{\zeta})=\frac{2 \ell+1}{4 \pi} \quad(\forall \boldsymbol{\zeta} \in \Sigma)
$$

Using the fact that

$$
\nabla Y_{\ell}^{m}(\boldsymbol{\zeta}) \cdot \nabla \bar{Y}_{\ell}^{m}(\boldsymbol{\zeta})=\frac{1}{2}\left[\Delta_{\Sigma}\left(Y_{\ell}^{m}(\boldsymbol{\zeta}) \bar{Y}_{\ell}^{m}(\boldsymbol{\zeta})\right)-\Delta_{\Sigma} Y_{\ell}^{m}(\boldsymbol{\zeta}) \bar{Y}_{\ell}^{m}(\boldsymbol{\zeta})-Y_{\ell}^{m}(\boldsymbol{\zeta}) \Delta_{\Sigma} \bar{Y}_{\ell}^{m}(\boldsymbol{\zeta})\right]
$$

together with (61) and the addition theorem (64), one also obtains

$$
\sum_{m=-\ell}^{+\ell} \nabla Y_{\ell}^{m}(\boldsymbol{\zeta}) \cdot \nabla \bar{Y}_{\ell}^{m}(\boldsymbol{\zeta})=\frac{\ell(\ell+1)(2 \ell+1)}{4 \pi} \quad(\forall \boldsymbol{\zeta} \in \Sigma) .
$$


The Jacobi-Anger expansion [1] for a plane wave propagating in direction $\delta \in \Sigma$ reads

$$
e^{\mathrm{i} k \boldsymbol{\zeta} \cdot \boldsymbol{\delta}}=\sum_{\ell=0}^{+\infty}(2 \ell+1) \mathrm{i}^{\ell} j_{\ell}(k|\boldsymbol{\zeta}|) P_{\ell}(\hat{\boldsymbol{\zeta}} \cdot \boldsymbol{\delta}) \quad\left(\forall \boldsymbol{\zeta} \in \mathbb{R}^{3}, \boldsymbol{\delta} \in \Sigma\right),
$$

where $P_{\ell}$ denotes the $\ell$-order Legendre polynomial. On deploying the addition theorem (63) in expansion (66), one obtains the Funk-Hecke formula

$$
\int_{\Sigma} e^{\mathrm{i} k \boldsymbol{\zeta} \cdot \boldsymbol{\delta}} Y_{\ell}^{m}(\boldsymbol{\delta}) \mathrm{d} \boldsymbol{\delta}=4 \pi \mathrm{i}^{\ell} j_{\ell}(k|\boldsymbol{\zeta}|) Y_{\ell}^{m}(\hat{\boldsymbol{\zeta}}) \quad\left(\forall \boldsymbol{\zeta} \in \mathbb{R}^{3}, \forall \ell \in \mathbb{N}, \forall m \in\{-\ell, \ldots, \ell\}\right) .
$$

The fundamental solution $G(\boldsymbol{\xi}, \boldsymbol{\zeta})$ of the Helmholtz equation admits the following expansion (Gegenbauer theorem [1]):

$$
G(\boldsymbol{\xi}, \boldsymbol{\zeta})=\frac{e^{\mathrm{i} k|\boldsymbol{\xi}-\boldsymbol{\zeta}|}}{4 \pi|\boldsymbol{\xi}-\boldsymbol{\zeta}|}=\frac{\mathrm{i} k}{4 \pi} \sum_{\ell=0}^{+\infty}(2 \ell+1) h_{\ell}(k|\boldsymbol{\zeta}|) j_{\ell}(k|\boldsymbol{\xi}|) P_{\ell}(\hat{\boldsymbol{\xi}} \cdot \hat{\boldsymbol{\zeta}}) \quad \text { if }|\boldsymbol{\zeta}|>|\boldsymbol{\xi}|
$$

which, using the addition theorem (63), can be recast in terms of spherical harmonics as

$$
G(\boldsymbol{\xi}, \boldsymbol{\zeta})=\mathrm{i} k \sum_{\ell=0}^{+\infty} \sum_{m=-\ell}^{+\ell} h_{\ell}(k|\boldsymbol{\zeta}|) j_{\ell}(k|\boldsymbol{\xi}|) Y_{\ell}^{m}(\hat{\boldsymbol{\xi}}) \bar{Y}_{\ell}^{m}(\hat{\boldsymbol{\zeta}}) \quad \text { if }|\boldsymbol{\zeta}|>|\boldsymbol{\xi}|
$$

\section{B Asymptotic behavior of special functions and their derivatives}

Spherical Bessel and Hankel functions of the first kind. The large-order asymptotic behavior of the spherical Bessel and Hankel functions $j_{n}$ and $h_{n}$ is [20]

$$
j_{n}(t)=t^{n} \frac{2^{n} n !}{(2 n+1) !}\left(1+O\left(n^{-1}\right)\right), \quad h_{n}(t)=\frac{1}{\mathrm{i} t^{n}} \frac{(2 n-1) !}{2^{n-1}(n-1) !}\left(1+O\left(n^{-1}\right)\right) \quad(n \rightarrow \infty) .
$$

Invoking the Stirling formula $n !=(n / e)^{n} \sqrt{2 \pi n}\left(1+O\left(n^{-1}\right)\right)$, one obtains the simpler asymptotic formulae

$$
j_{n}(t) \underset{n \rightarrow \infty}{=} \frac{1}{2 n \sqrt{2}}\left(\frac{e t}{2 n}\right)^{n}\left(1+O\left(n^{-1}\right)\right), \quad h_{n}(t) \underset{n \rightarrow \infty}{=} \frac{\sqrt{2}}{\mathrm{i} t}\left(\frac{2 n}{e t}\right)^{n}\left(1+O\left(n^{-1}\right)\right)
$$

Moreover, the following small-argument and large-argument asymptotic expressions of $j_{n}$ are also available:

$$
\begin{array}{ll}
\left.j_{n}(t) \underset{t \rightarrow 0}{=} \frac{t^{n}}{(2 n+1) ! !}(1+O(t))\right), & \left.h_{n}(t) \underset{t \rightarrow 0}{=} \mathrm{i} \frac{(2 n-1) ! !}{t^{n}}(1+O(t))\right), \\
j_{n}(t) \underset{t \rightarrow \infty}{=} \frac{1}{t} \cos \left[t-\frac{n+1}{2} \pi\right]\left(1+O\left(t^{-1}\right)\right) . & h_{n}(t) \underset{t \rightarrow \infty}{=} \mathrm{i}^{-n-1} e^{\mathrm{i} t} \frac{1}{t}
\end{array}
$$

(with $(2 n+1) ! !:=1 \times 3 \times \ldots(2 n+1)$ for any integer $n \geq 0)$. 
Derivatives of spherical Bessel and Hankel functions. Since the derivatives $j_{n}^{\prime}$ and $h_{n}^{\prime}$ are given by the recurrence relation [1]

$$
f_{n}^{\prime}(t)=\frac{n f_{n-1}(t)-(n+1) f_{n+1}(t)}{2 n+1} \quad\left(f_{n}=j_{n} \text { or } h_{n}\right) \quad(n \geq 1)
$$

the following large-order asymptotic formulae are readily deduced from (70) as

$$
j_{n}^{\prime}(t) \underset{n \rightarrow \infty}{=} \frac{1}{2 t \sqrt{2}}\left(\frac{e t}{2 n}\right)^{n}\left(1+O\left(n^{-1}\right)\right), \quad h_{n}^{\prime}(t) \underset{n \rightarrow \infty}{=} \frac{-n \sqrt{2}}{i t^{2}}\left(\frac{2 n}{e t}\right)^{n}\left(1+O\left(n^{-1}\right)\right)
$$

Moreover, the following small-argument and large-argument asymptotic expressions of $j_{n}^{\prime}$ are obtained by applying (73) to (71) and (72)

$$
\begin{aligned}
& \left.\left.j_{n}^{\prime}(t) \underset{t \rightarrow 0}{=} \frac{n t^{n-1}}{(2 n+1) ! !}(1+O(t))\right), \quad h_{n}^{\prime}(t) \underset{t \rightarrow 0}{=}-\mathrm{i} \frac{(n+1)(2 n-1) ! !}{t^{n+1}}(1+O(t))\right) \\
& j_{n}^{\prime}(t) \underset{t \rightarrow \infty}{=} \frac{1}{t} \cos \left[t-\frac{n}{2} \pi\right]\left(1+O\left(t^{-1}\right)\right), \frac{4 \pi}{R^{2 \ell}}
\end{aligned}
$$

whereas for the special case $n=0$ one has

$$
\left.\left.j_{0}^{\prime}(t) \underset{t \rightarrow 0}{=}-\frac{t}{3}(1+O(t))\right), \quad h_{0}^{\prime}(t) \underset{t \rightarrow 0}{=} \frac{\mathrm{i}}{t^{2}}(1+O(t))\right), \quad j_{0}^{\prime}(t) \underset{t \rightarrow \infty}{=} \frac{\cos t}{t}\left(1+O\left(t^{-1}\right)\right) .
$$

\section{References}

[1] Abramowitz, M., Stegun, I. A. Handbook of mathematical functions, with formulas, graphs, and mathematical tables. Dover (1965).

[2] Ammari, H., Garnier, J., Jugnon, V., Kang, H. Stability and resolution analysis for a topological derivative based imaging functional. SIAM J. Contr. Opt., 50:48-76 (2012).

[3] Ammari, H., Garnier, J., Kang, H., Lim, M., Sølna, K. Multistatic Imaging of Extended Targets. SIAM J. Imag. Sci., 5(2):564-600 (2012).

[4] Ammari, H, Iakovleva, E., Lesselier, D., Perrusson, G. MUSIC-type electromagnetic imaging of a collection of small three-dimensional inclusions. SIAM J. Sci. Comput., 29:674-709 (2007).

[5] Ammari, H., Kang, H. Reconstruction of the small inhomogeneities from boundary measurements. Springer, Berlin, New York (2004).

[6] Ammari, H., Kang, H. Polarization and moment tensors with applications to inverse problems and effective medium theory. Springer (2007).

[7] Arens, T., Lechleiter, A., Luke, D.R. MUSIC for Extended Scatterers as an Instance of the Factorization Method. SIAM J. Appl. Math., 70:1283-1304 (2009).

[8] Bonnet, M. BIE and material differentiation applied to the formulation of obstacle inverse problems. Eng. Anal. with Bound. Elem., 15:121-136 (1995).

[9] Bonnet, M. Topological sensitivity for 3D elastodynamic and acoustic inverse scattering in the time domain. Comput. Methods Appl. Mech. Engrg, 195:5239-5254 (2006).

[10] Bonnet, M., Guzina, B. B. Sounding of finite solid bodies by way of topological derivative. Int. J. Numer. Meth. Engng, 61:2344-2373 (2004).

[11] Bourgeois, L., Luneville, E. On the use of sampling methods to identify cracks in acoustic waveguides. Inverse Problems, 28:105011 (2012). 
[12] Brandfass, M, Lanterman, A D, Warnick, K F. A comparison of the Colton-Kirsch inverse scattering methods with linearized tomographic inverse scattering. Inverse Problems, 17:1797-1816 (2001).

[13] Cakoni, F., Colton, D. Qualitative Methods in Inverse Scattering Theory. Springer-Verlag Berlin (2006).

[14] Carpio, A., Rapun, M. L. Solving inhomogeneous inverse problems by topological derivative methods. Inverse Problems, 24:045014 (2008).

[15] Cedio-Fengya, D. J., Moskow, S., Vogelius, M. S. Identification of conductivity imperfections of small diameter by boundary measurements. Continuous dependence and computational reconstruction. Inverse Problems, 14:553-595 (1998).

[16] Cheney, M. The linear sampling method and the MUSIC algorithm. Inverse Problems, 17:591-596 (2001).

[17] Collino, F., Fares, M. B., Haddar, H. Numerical and analytical studies of the linear sampling method in electromagnetic inverse scattering problems. Inverse problems, 19:12791298 (2003).

[18] Colton, D., Coyle, J., Monk, P. Recent developments in inverse acoustic scattering theory. SIAM Review, 42:369-414 (2000).

[19] Colton, D., Kirsch, A. A simple method for solving inverse scattering problems in the resonance region. Inverse Problems, 12:383-393 (1996).

[20] Colton, D., Kress, R. Inverse acoustic and electromagnetic scattering theory. SpringerVerlag (1992).

[21] Colton, D., Monk, P. The inverse scattering problem for time-harmonic acoustic waves in a penetrable medium. Quart. J. Mech. Appl. Math., 40:189-212 (1987).

[22] Colton, D., Päivärinta, L., Sylvester, J. The interior transmission problem. Inverse Problems and Imaging, 1:13-28 (2007).

[23] Dassios, G., Kleiman, R. Low frequency scattering. Oxford Science Publications (2000).

[24] Feijoo, G. R. A new method in inverse scattering based on the topological derivative. Inverse Problems, 20:1819-1840 (2004).

[25] Fessenden, R. Method and Apparatus for Finding Ore Bodies. US patent 1240328, USA (18 Sept. 1917).

[26] Gerlach, T., Kress, R. Uniqueness in inverse obstacle scattering with conductive boundary condition. Inverse Problems, 12:619-625 (1996).

[27] Guzina, B. B., Bonnet, M. Small-inclusion asymptotic of misfit functionals for inverse problems in acoustics. Inverse Problems, 22:1761-1785 (2006).

[28] Guzina, B. B., Chikichev, I. From imaging to material identification: a generalized concept of topological sensitivity. J. Mech. Phys. Solids, 55:245-279 (2007).

[29] Guzina, B. B., Madyarov, A. I. A linear sampling approach to inverse elastic scattering in piecewise-homogeneous domains. Inverse Problems, 23:1467-1493 (2006).

[30] Kinsler, L. E., Frey, A. R., Coppens, A. B., Sanders, J. V. Fundamentals of acoustics. John Wiley and Sons (1982).

[31] Kirsch, A. On the existence of transmission eigenvalues. Inverse Problems and Imaging, 3:155-172 (2009).

[32] Kirsch, A., Grinberg, N. The Factorization Method for Inverse Problems. Oxford University Press, Oxford, UK (2007). 
[33] Kirsch, A., Kress, R. Uniqueness in inverse obstacle scattering (acoustics). Inverse Problems, 9:285-299 (1993).

[34] Kress, R. Linear integral equations. Springer (1999).

[35] Malcolm, A., Guzina, B. B. On the topological sensitivity of transient acoustic fields. Wave Motion, 45:821-834 (2008).

[36] Masmoudi, M., Pommier, J., Samet, B. The topological asymptotic expansion for the Maxwell equations and some applications. Inverse Problems, 21:547-564 (2005).

[37] McLean, W. Strongly elliptic systems and boundary integral equations. Cambridge University Press (2000).

[38] Nachman, A.I. Reconstructions from boundary measurements. Annals Math., 128:531-576 (1988).

[39] Nagayasu, S., Uhlmann, G., Wang, J.-N. Reconstruction of penetrable obstacles in acoustic scattering. SIAM J. Math. Anal., 43:189-211 (2011).

[40] Nédélec, J.-C. Acoustic and electromagnetic equations. Integral representations for harmonic problems. Springer (2001).

[41] Nintcheu Fata, S., Guzina, B. B. A linear sampling method for near-field inverse problems in elastodynamics. Inverse Problems, 20:713-736 (2004).

[42] Päivärinta, L., Sylvester, J. Transmission eigenvalues. SIAM J. Math. Anal., 40:738-753 (2008).

[43] Pike, R., Sabatier, P. (eds.). Scattering: Scattering and Inverse Scattering in Pure and Applied Science, vol. 1. Academic Press, San Diego (2002).

[44] Potthast, R. Point Sources and Multipoles in Inverse Scattering Theory. Chapman and Hall / CRC, New York (2001).

[45] Potthast, R. A survey on sampling and probe methods for inverse problems. Inverse Problems, 22:R1-R47 (2006).

[46] Roy, D.N. Ghosh, Warner, J.A., Couchman, L.S., Shirron, J. Inverse obstacle transmission problem in acoustics. Inverse Problems, 14:923-929 (1998).

[47] Schmidt, R. Multiple emitter location and signal parameter estimation. IEEE Trans. Antenn. Prop., 34:276-280 (1986).

[48] Sokolowski, J., Zochowski, A. On the topological derivative in shape optimization. SIAM J. Control Optim., 37:1251-1272 (1999).

[49] Tarantola, A. A strategy for nonlinear elastic inversion of seismic reflection data. Geophysics, 51:1893-1903 (1986).

[50] Warner, J.A., Roy, D.N. Ghosh, Bucaro, J., Couchman, L. Inversion of penetrable obstacles from far-field data on narrow angular apertures. J. Acoust. Soc. Am., 107:1111-1120 (2000).

[51] Xiaodong, L., Bo, Z. Inverse scattering by an inhomogeneous penetrable obstacle in a piecewise homogeneous medium. Acta Math. Scientia, 32:1281-1297 (2012). 